\title{
ABJ theory in the higher spin limit
}

\author{
Shinji Hirano, ${ }^{a}$ Masazumi Honda, ${ }^{b, 1}$ Kazumi Okuyama $^{c}$ and Masaki Shigemori ${ }^{d, e}$ \\ ${ }^{a}$ School of Physics and Mandelstam Institute for Theoretical Physics, \\ and DST-NRF Centre of Excellence in Mathematical and Statistical Sciences (CoE-MaSS), \\ University of the Witwatersrand, \\ WITS 2050, Johannesburg, South Africa \\ ${ }^{b}$ Harish-Chandra Research Institute, \\ Chhatnag Road, Jhusi, Allahabad 211019, India \\ ${ }^{c}$ Department of Physics, Shinshu University, \\ Matsumoto 390-8621, Japan \\ ${ }^{d}$ Yukawa Institute for Theoretical Physics, Kyoto University, \\ Kitashirakawa Oiwakecho, Sakyo-ku, Kyoto 606-8502 Japan \\ ${ }^{e}$ Hakubi Center, Kyoto University, \\ Yoshida-Ushinomiya-cho, Sakyo-ku, Kyoto 606-8501, Japan \\ E-mail: shinji.hirano@wits.ac.za, masazumi.honda@weizmann.ac.il, \\ kazumi@azusa.shinshu-u.ac.jp, shige@yukawa.kyoto-u.ac.jp
}

Abstract: We study the conjecture made by Chang, Minwalla, Sharma, and Yin on the duality between the $\mathcal{N}=6$ Vasiliev higher spin theory on $\mathrm{AdS}_{4}$ and the $\mathcal{N}=6$ Chern-Simons-matter theory, so-called ABJ theory, with gauge group $\mathrm{U}(N) \times \mathrm{U}(N+M)$. Building on our earlier results on the ABJ partition function, we develop the systematic $1 / M$ expansion, corresponding to the weak coupling expansion in the higher spin theory, and compare the leading $1 / M$ correction, with our proposed prescription, to the one-loop free energy of the $\mathcal{N}=6$ Vasiliev theory. We find an agreement between the two sides up to an ambiguity that appears in the bulk one-loop calculation.

Keywords: AdS-CFT Correspondence, Chern-Simons Theories, Higher Spin Gravity, Supersymmetric gauge theory

ARXIV EPRINT: 1504.00365

\footnotetext{
${ }^{1}$ The current affiliation: Department of Particle Physics and Astrophysics, Weizmann Institute of Science, Rehovot 7610001, Israel.
} 


\section{Contents}

1 Introduction 1

2 The main results 3

3 The boundary side: ABJ theory $\quad 6$

3.1 The ABJ partition function 6

3.2 The large $M$ expansion 8

4 The bulk side: $\mathcal{N}=6$ Vasiliev theory 10

$\begin{array}{lll}4.1 & \text { The one-loop contribution } & 11\end{array}$

$\begin{array}{lll}4.2 & \text { The bosonic contributions } & 14\end{array}$

$\begin{array}{ll}4.3 \text { The fermionic contributions } & 15\end{array}$

$\begin{array}{ll}4.4 \text { The full one-loop free energy } & 16\end{array}$

$\begin{array}{llr}5 & \text { Discussions } & 17\end{array}$

$\begin{array}{lr}\text { A Formal expansion of } f(x, k, t) & 19\end{array}$

B Evaluation of the matrix integral (3.23) 24

\section{Introduction}

It has long been speculated that string theory in the high energy limit $E \sqrt{\alpha^{\prime}} \rightarrow \infty$ undergoes drastic reduction of degrees of freedom due presumably to enhanced symmetries associated with an infinite number of massless fields which appear in this limit [1-3]. This is the extremity of stringy regime and may reveal what string theory truly is. The infinite number of massless fields are higher spin fields, and the high energy limit of string theory may thus yield higher spin (HS) theory. String theory might then be realized as the symmetry broken phase of HS theory where the mass scale $1 / \sqrt{\alpha^{\prime}}$ is dynamically generated.

Higher spin theory has generated a great deal of interest recently. This goes back to the old work of Vasiliev [4-8] who constructed interacting theories of massless higher spin fields that successfully included gravity, i.e., a spin-2 field. The crucial idea was to consider HS theories on de Sitter (dS) or anti-de Sitter (AdS) space, instead of Minkowski space, in order to evade no-go theorems concerning massless higher spin fields [9-13]. Years later, Klebanov and Polyakov [14] made the important conjecture that the HS theory on $\mathrm{AdS}_{4}$ space is dual to the $O(N)$ vector model (VM) at critical points. Substantial and highly nontrivial evidence for the HS/VM duality was later provided by Giombi and Yin who demonstrated that 3-point functions of conserved higher spin currents agree on both sides $[15,16]$. This conjecture and its generalizations were further tested successfully at 
one loop of the HS theory for the vector models at both UV and IR fixed points [17-20]. Meanwhile, the collective field method was applied to the vector models, elucidating how the HS theory can be directly reconstructed from the VM as well as providing a new perspective on the origin of the duality as a gauge phenomenon [21-26]. It should also be noted that, pioneered by Gaberdiel and Gopakumar, tremendous progress has been made in the study of the duality between HS theories on $\mathrm{AdS}_{3}$ and minimal $\mathrm{CFT}_{2}$ 's due to the relative simplicity in lower dimensionality $[27-51]$.

String theory on AdS space in the limit $\sqrt{\alpha^{\prime}} / R_{\mathrm{AdS}} \rightarrow \infty$ may provide a concrete example in which one can probe the symmetric phase of string theory in the high energy limit and study its connection to HS theory. ${ }^{1}$ Via the AdS/CFT correspondence, the limit may also give us the vector model dual to the HS theory. Indeed, such an example was suggested by Chang, Minwalla, Sharma, and Yin (CMSY) [54] who proposed the HS limit of $\mathrm{AdS}_{4} / \mathrm{CFT}_{3}$ with $\mathcal{N}=6$ supersymmetries (SUSY), the version conjectured by Aharony, Bergman, and Jafferis (ABJ) [55] that generalized their earlier work with Maldacena (ABJM) [56]. The gravity theory is M-theory on $\mathrm{AdS}_{4} \times S^{7} / \mathbb{Z}_{k}$ with the 3 -form field turned on, $C_{3} \propto M$, and the dual field theory is the $\mathcal{N}=6 \mathrm{U}(N)_{k} \times \mathrm{U}(N+M)_{-k}$ Chern-Simons-matter (CSM) theory, called the ABJ theory, where $k$ and $-k$ are the ChernSimons levels for the two gauge groups. At large $k$, the M-theory circle of radius $R_{11}=1 / k$ shrinks and M-theory reduces to type IIA string theory on $\mathrm{AdS}_{4} \times \mathbb{C P}^{3}$ with the NSNS 2 -form turned on, $B_{2} \propto \frac{M}{k}-\frac{1}{2}[55,57,58]$. The ingredient crucial to the HS/VM duality is the presence of the $B_{2}$ that, in particular, provides $\mathrm{U}(M)$ vectors in the dual field theory. The HS limit proposed by CMSY is

$$
M,|k| \longrightarrow \infty \quad \text { with } t \equiv \frac{M}{|k|} \text { and } \quad N \quad \text { finite }
$$

which is conjectured to be the $\mathcal{N}=6 \mathrm{U}(N)$ Vasiliev theory, constructed by CMSY and Sezgin-Sundell $[59,60]$, where the Newton constant $G_{\mathrm{HS}}$ of the HS theory is proportional to $1 / M,{ }^{2}$ and the parity-violating (PV) phase $\theta_{0}=\pi t / 2$. This is, in fact, the high energy limit of type IIA string theory, since the string length is large, $\sqrt{\alpha^{\prime}} / R_{\text {AdS }} \sim(k / N)^{1 / 4} \rightarrow \infty$. As a comparison, let us consider type IIB string theory on $\operatorname{AdS}_{5} \times S^{5}$. If we take the $\sqrt{\alpha^{\prime}} / R_{\mathrm{AdS}}^{\mathrm{IIB}} \rightarrow \infty$ limit, the 't Hooft coupling $\lambda \rightarrow 0$ and the dual field theory, $\mathcal{N}=4$ super Yang-Mills (SYM) theory, becomes free. ${ }^{3}$ This is in contrast with the ABJ theory which remains nontrivial in the high energy limit (1.1).

Therefore, the ABJ theory in the HS limit is an ideal setup to study the high energy regime of string theory and elucidate its non-trivial dynamics. In this paper we study the HS limit of CMSY by (1) developing the systematic $1 / M$ expansion of the free energy of the ABJ theory, (2) calculating the one-loop free energy of the $\mathcal{N}=6$ HS theory, and (3) subjecting the results to a one-loop test.

\footnotetext{
${ }^{1}$ In the case of the HS theory on $\mathrm{AdS}_{3}$ with $\mathcal{N}=4$ supersymmetries it was shown via the AdS/CFT correspondence that the HS theory describes a closed subsector in the symmetric phase of the type IIB string theory on $\mathrm{AdS}_{3} \times S^{3} \times T^{4}$ in the high energy limit $[52,53]$.

${ }^{2}$ In CMSY, the Newton constant $G_{\mathrm{HS}}$ was identified with $\frac{1}{M+N}$. However, as we will see below, the finite $M$ corrections instead suggest that the identification $G_{\mathrm{HS}} \propto \frac{1}{M}$ works better.

${ }^{3}$ It should be noted that there has been significant progress in the study of the free field limit of $\mathrm{AdS}_{5} / \mathrm{CFT}_{4}[61-67]$.
} 
The free energy or the partition function of the ABJ $(M)$ theory has been studied extensively over the last few years thanks to the localization technique [68] which drastically simplifies path integrals of supersymmetric gauge theories [69, 70]. Inspired by the seminal work of Drukker, Marino, and Putrov [71, 72] and, in good part, with the use of the elegant Fermi gas approach developed by Marino and Putrov [73], a great deal about the $\mathrm{ABJ}(\mathrm{M})$ partition function has been uncovered, in particular, at large $N$, both in perturbative $[73,74]$ and nonperturbative expansions [75-82]. There has also been significant progress in the study of Wilson loops in the ABJ(M) theory [83-86] as well as the partition functions of more general Chern-Simons-matter theories [87-91]. However, the ABJ partition function in the HS limit (1.1) has not been much investigated in the literature. In the current paper, building on our earlier work [92, 93], we develop a systematic procedure to compute a large $M$ expansion of the partition function and start exploring the highly stringy regime of the HS/ABJ duality at finite $N$. The HS limit can alternatively be extracted from the conifold expansion developed in [94], but our approach has the advantage of directly giving the $1 / M$ expansion. ${ }^{4}$

To compare the $1 / M$ expansion of the ABJ free energy with that of the HS free energy, an obstacle is the lack of the action for the Vasiliev theory from which to extract a weak coupling expansion. ${ }^{5}$ In this paper, following refs. [17, 20], we circumvent this problem by computing the one-loop free energy, which can be computed without the action as long as we know the spectrum, and by comparing it with the ABJ free energy. In specifying the spectrum, it is crucial to choose appropriate boundary conditions of the HS fields. For generic $t$, however, nontrivial boundary conditions bring technical difficulties into the calculation. For this reason, we adopt the strategy that performing the calculation in the regime $t \ll 1$ and then, with the help of the result in [95], we infer the form of the one-loop free energy for generic $t$.

The organization of this paper is as follows: in section 2 we summarize our claim and the main results on the HS and ABJ free energy and the correspondence between the two sides. In section 3 we review the integral representation, sometimes referred to as "mirror description" of the ABJ partition function, using which we analyze the free energy in the HS limit and develop a systematic $1 / M$ expansion. Some of the technical details in section 3 are provided in appendices A and B. In section 4 we calculate the one-loop free energy of $\mathcal{N}=6$ Vasiliev HS theory. We close our paper with discussions in section 5 .

\section{The main results}

We first summarize our claim and the main results on the correspondence between the $\mathcal{N}=6$ HS and ABJ free energies in the limit (1.1) with $1 / M$ corrections.

Higher spin theories are dual to vector models. Our working assumption is that the vector degrees of freedom dual to the $\mathcal{N}=6$ HS theory are massless open strings stretched between $N$ regular and $M$ fractional D3-branes in the type IIB frame of the (UV-completed)

\footnotetext{
${ }^{4}$ We thank Marcos Mariño for pointing out to us the use of the conifold expansion for the HS limit.

${ }^{5}$ Although there are some propositions about actions of the Vasiliev theory [96-99], it is not obvious to compute tree level free energy from these actions.
} 


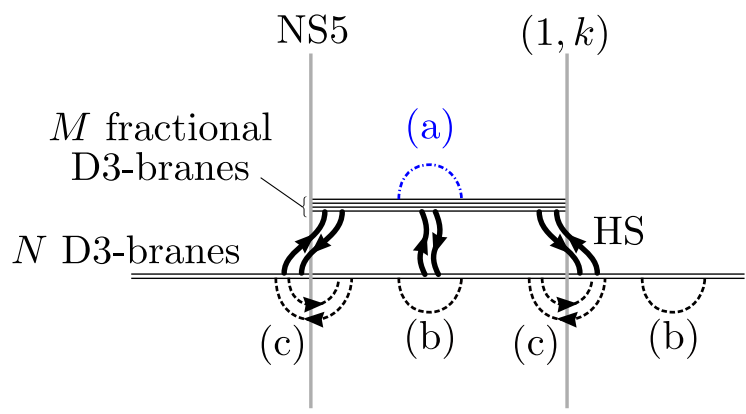

Figure 1. The open-string interpretation of the field content of the ABJ theory in the type IIB UV description. $N$ D3-branes are intersecting with an NS5-brane and with a $(1, k) 5$-brane, and wrap the horizontal direction which is periodically identified. $M$ fractional D3-branes partially wrap the horizontal direction, ending on the 5-branes. (For more detail about the brane configuration, see $[55,56]$.) The open strings stretching between D3-branes represent fields in the ABJ theory. To obtain the fields relevant for the duality to higher spin (HS) theory, we must remove the open strings related to the $\mathrm{U}(M)$ CS theory ((a), blue dashed-dotted line). The HS degrees of freedom are dual to combinations of $\mathrm{U}(M)$ vectors (thick black lines), $\mathrm{U}(N)$ adjoints (b) and $\mathrm{U}(N) \times \mathrm{U}(N)$ bi-fundamentals (c) (black dashed lines).

ABJ theory; see figure 1. Since the ABJ theory has a $\mathrm{U}(N) \times \mathrm{U}(N+M)$ adjoint and $(\bar{N}, N+M)$ bi-fundamentals with their conjugates, in addition to the $\mathrm{U}(M)$ vectors which are expected to be dual to the higher spin fields, we have non-vector degrees of freedom, i.e., (a) the $\mathrm{U}(M)$ adjoint, (b) $\mathrm{U}(N) \times \mathrm{U}(N)$ adjoints, (c) the $(\bar{N}, N)$ bi-fundamentals and their conjugates. Note that (b) and (c) give the same matter content as that appears in the $\mathrm{U}(N)_{k} \times \mathrm{U}(N)_{-k}$ ABJM theory.

As alluded in the introduction, we wish to compare the free energy (or the partition function) of the $\mathcal{N}=6 \mathrm{U}(N)$ Vasiliev HS theory with the coupling $G_{\mathrm{HS}}$ and the PV phase $\theta_{0}$ with that of the $\mathrm{U}(N)_{k} \times \mathrm{U}(N+M)_{-k}$ ABJ theory. Since the $\mathrm{U}(M)$ adjoint fields are clearly unwanted degrees of freedom, they have to be removed in the HS/ABJ duality. We thus propose that the partition function $Z_{\mathrm{HS}}\left(G_{\mathrm{HS}}, \theta_{0} ; N\right)$ of the former, normalized by the $\mathrm{U}(N)$ volume, is related to that of the latter, $Z_{\mathrm{ABJ}}(N, N+M)_{k}$, by the quotient ${ }^{6}$

$$
\frac{Z_{\mathrm{HS}}\left(G_{\mathrm{HS}}, \theta_{0} ; N\right)}{\operatorname{Vol}(\mathrm{U}(N))}=\frac{\left|Z_{\mathrm{ABJ}}(N, N+M)_{k}\right|}{Z_{\mathrm{CS}}(M)_{k}}
$$

with the identification of the parameters ${ }^{7}$

$$
G_{\mathrm{HS}}=\frac{\gamma}{M} \frac{\pi t}{\sin (\pi t)} \quad \text { and } \quad \theta_{0}=\frac{\pi t}{2},
$$

\footnotetext{
${ }^{6}$ We revise the proposal in the previous version of our paper,

$$
Z_{\mathrm{HS}}\left(G_{\mathrm{HS}}, \theta_{0} ; N\right)=\frac{1}{\operatorname{Vol}(\mathrm{U}(N))} \frac{\left|Z_{\mathrm{ABJ}}(N, N+M)_{k}\right|}{Z_{\mathrm{CS}}(M)_{k} Z_{\mathrm{ABJM}}(N)_{k}}
$$

which we believe was incorrect.

${ }^{7}$ More recently, one of the authors determined the constant $\gamma$ to be $\gamma=\frac{2}{\pi}$ by computing the two point function of the stress-energy tensor [100].
} 
where $\gamma$ is a constant that cannot be fixed by the analysis of the current paper, $t=M /|k|$ as defined in (1.1), and $Z_{\mathrm{CS}}(M)_{k}$ is the partition function of the $\mathcal{N}=2 \mathrm{U}(M)$ Chern-Simons theory at level $k$.

As indicated in figure 1, the (massless) open strings involved in (2.2) are $\mathrm{U}(M)$ vectors and $\mathrm{U}(N) \times \mathrm{U}(N)$ bi-fundamentals and adjoints. The HS fields, which are $\mathrm{U}(N)$ adjoints, arise by connecting these open strings as follows. Among the open strings, there are two types of $\mathrm{U}(M)_{-k}$ vectors, namely (i) the $\mathrm{U}(M)_{-k} \times \mathrm{U}(N)_{-k}$ bi-fundamentals which are contained in the $\mathrm{U}(N+M)_{-k}$ adjoint and represented in figure 1 by the middle pair of black thick arrows, and (ii) the $\mathrm{U}(M)_{-k} \times \mathrm{U}(N)_{k}$ bi-fundamentals which are represented in figure 1 by the pairs of black thick arrows on the right and left. Each of these $\mathrm{U}(M)_{-k^{-}}$ vector strings can be connected with the $\mathrm{U}(N)_{-k} \times \mathrm{U}(N)_{k}$ bi-fundamentals, open strings (c), to form (i) $\mathrm{U}(M)_{-k} \times \mathrm{U}(N)_{k}$ bi-fundamentals and (ii) $\mathrm{U}(M)_{-k} \times \mathrm{U}(N)_{-k}$ bi-fundamentals. The latter bi-fundamental strings can be further connected with their conjugates on their $\mathrm{U}(M)_{-k}$ endpoints to form (i) $\mathrm{U}(N)_{k}$ and (ii) $\mathrm{U}(N)_{-k}$ adjoints. These $\mathrm{U}(N)$ adjoints correspond to the HS fields with pure, as opposed to mixed, boundary conditions. On the other hand, the $\mathrm{U}(N)_{k}$ and $\mathrm{U}(N)_{-k}$ adjoints represented by open strings (b) correspond to spin 1 fields with the mixed boundary condition. (The latter would have been absent if the $\mathrm{U}(N)$ symmetries were not gauged.)

The identification of the Newton constant $G_{\mathrm{HS}}$ in (2.3) can be inferred from the $1 / M$ expansion (3.25) of the ABJ free energy in which $1 / M$ systematically appears in the combination $G_{\mathrm{HS}}$. The proposal (2.2) then predicts the HS free energy, $F_{\mathrm{HS}} \equiv-\ln Z_{\mathrm{HS}}$, to be ${ }^{8}$

$$
\begin{aligned}
F_{\mathrm{HS}}\left(G_{\mathrm{HS}}, \theta_{0}, N\right)= & \frac{\gamma N}{G_{\mathrm{HS}}} \frac{2 \mathcal{I}\left(\theta_{0}\right)}{\sin \left(2 \theta_{0}\right)}+\frac{N^{2}}{2} \ln \left(\frac{2 \gamma}{\pi G_{\mathrm{HS}}}\right)-\frac{N^{2}}{2} \ln \left(\sin ^{2}\left(2 \theta_{0}\right)\right) \\
& -\left(2 N^{2}-1\right)\left(3 \cos \left(4 \theta_{0}\right)+1\right) \frac{N G_{\mathrm{HS}}}{48 \gamma}+\mathcal{O}\left(G_{\mathrm{HS}}^{2}\right)
\end{aligned}
$$

where

$$
\mathcal{I}(x) \equiv-\int_{0}^{x} d y \ln \tan y=\operatorname{Im}\left[\operatorname{Li}_{2}(i \tan x)\right]-x \ln \tan x=\mathcal{I}\left(\frac{\pi}{2}-x\right) .
$$

It is worth emphasizing that the Newton constant $G_{\mathrm{HS}}$ agrees with the one suggested by the computation of three point functions of higher spin currents for non-supersymmetric theories which is an independent and a completely different analysis [101]. Furthermore, as remarked in footnote 7 , the constant $\gamma$ has been recently determined to be $\gamma=2 / \pi$ in [100] from the two point function of the stress-energy tensor.

The proposal (2.2) was motivated in part to respect the invariance under the duality

$$
M \leftrightarrow|k|-M, \quad k \leftrightarrow-k,
$$

which can be expressed in terms of the HS parameter as

$$
\theta_{0} \rightarrow \frac{\pi}{2}-\theta_{0}
$$

\footnotetext{
${ }^{8}$ With the large $M$ expansion we develop in section 3 , one can in principle compute the expansion to arbitrary finite order. In eq. (3.25), we present the explicit expansion up to order $G_{\mathrm{HS}}^{4} \propto 1 / M^{4}$ terms.
} 
In the case of the ABJ theory this is known as the Giveon-Kutasov-Seiberg duality under which the partition function $Z_{\mathrm{ABJ}}(N, N+M)_{k}$ is invariant [55, 102]. For the CS partition function $Z_{\mathrm{CS}}(M)_{k}$, this is nothing but the level-rank duality. Note that the Newton constant $G_{\mathrm{HS}}$ in (2.3) is a duality invariant.

The HS free energy (2.4) has a few favorable features: (1) The leading $1 / G_{\mathrm{HS}}$ term is linear in $M$, as opposed to $M^{2}$ as would be expected from the $\mathrm{U}(M)$ vector degrees of freedom, and the dependence on the PV phase $\theta_{0}$ is qualitatively similar to that of the $\mathcal{N}=2$ theory in [103] which exhibits the invariance under $\theta_{0} \leftrightarrow \frac{\pi}{2}-\theta_{0}$. (2) The leading $1 / M$ correction, the first logarithmic term in (2.4), is consistent with the one-loop free energy of the $\mathcal{N}=6$ HS theory whose contribution comes solely from the $\mathrm{U}(N)$ gauge fields, as calculated in section 4 , up to the ambiguity of the constant $\gamma$.

Finally, the presence of the third term $-\frac{N^{2}}{2} \ln \left(\sin ^{2}\left(2 \theta_{0}\right)\right)$ in (2.4) may call for a further explanation. This is a part of the HS one-loop contribution and diverges logarithmically as the PV phase $\theta_{0}$ is switched off or takes the maximal value $\pi / 2 .{ }^{9}$ Although this might look like an unpleasant result, it can be argued that this indeed precisely agrees with the $\tilde{\lambda}$ dependent factor in the anomalous dimension eq. (A.5) of [95] predicted from HS symmetry considerations. We will make a more detailed discussion on this point later in section 5 .

\section{The boundary side: ABJ theory}

In this section, we study the HS limit of the partition function of the ABJ theory and develop a systematic way to derive its large $M$ expansion. The expansion can be explicitly worked out any finite order in principle. In the next section, we will use the 1-loop part of the expansion for comparison with the bulk Vasiliev theory.

\subsection{The ABJ partition function}

The partition function of the $\mathrm{U}\left(N_{1}\right)_{k} \times \mathrm{U}\left(N_{2}\right)_{-k}$ ABJ theory on $S^{3}$ has been written in the matrix model form $[68,69]$ using the localization technique [70]. The explicit expression of the partition function is

$$
Z_{\mathrm{ABJ}}\left(N_{1}, N_{2}\right)_{k}=\mathcal{N} \int \prod_{j=1}^{N_{1}} \frac{d \mu_{j}}{2 \pi} \prod_{a=1}^{N_{2}} \frac{d \nu_{a}}{2 \pi} \frac{\Delta_{\mathrm{sh}}(\mu)^{2} \Delta_{\mathrm{sh}}(\nu)^{2}}{\Delta_{\mathrm{ch}}(\mu, \nu)^{2}} e^{\frac{i k}{4 \pi}\left(\sum_{j=1}^{N_{1}} \mu_{j}^{2}-\sum_{a=1}^{N_{2}} \nu_{a}^{2}\right)},
$$

where $\Delta_{\mathrm{sh}}$ and $\Delta_{\mathrm{ch}}$ are the one-loop determinant of the vector multiplets and the matter multiplets in the bi-fundamental representation, respectively:

$$
\begin{aligned}
\Delta_{\mathrm{sh}}(\mu) & =\prod_{1 \leq j<m \leq N_{1}}\left(2 \sinh \frac{\mu_{j}-\mu_{m}}{2}\right), \quad \Delta_{\mathrm{sh}}(\nu)=\prod_{1 \leq a<b \leq N_{2}}\left(2 \sinh \frac{\nu_{a}-\nu_{b}}{2}\right) \\
\Delta_{\mathrm{ch}}(\mu, \nu) & =\prod_{j=1}^{N_{1}} \prod_{a=1}^{N_{2}}\left(2 \cosh \frac{\mu_{j}-\nu_{a}}{2}\right) .
\end{aligned}
$$

\footnotetext{
${ }^{9}$ In fact, the first term in (2.4) which is the classical contribution also diverges logarithmically as $\theta_{0} \rightarrow 0$ or $\pi / 2$. With the lack of full understanding of the HS theory action, it is not clear how this singularity should be interpreted.
} 
Furthermore, $k \in \mathbb{Z}_{\neq 0}$ is the Chern-Simons level, while $\mathcal{N}$ is the normalization factor [71, 72]

$$
\mathcal{N} \equiv \frac{i^{-\frac{\kappa}{2}\left(N_{1}^{2}-N_{2}^{2}\right)}}{N_{1} ! N_{2} !}, \quad \kappa \equiv \operatorname{sign} k .
$$

Because of the relation

$$
Z_{\mathrm{ABJ}}\left(N_{2}, N_{1}\right)_{k}=Z_{\mathrm{ABJ}}\left(N_{1}, N_{2}\right)_{-k}=Z_{\mathrm{ABJ}}\left(N_{1}, N_{2}\right)_{k}^{*},
$$

we can assume $N_{1} \leq N_{2}$ and $k>0$ without loss of generality, as we will do henceforth. We set

$$
N_{1} \equiv N, \quad N_{2} \equiv N+M, \quad M \geq 0 .
$$

We write $Z_{\mathrm{ABJ}}\left(N_{1}, N_{2}\right)$ also as $Z_{\mathrm{ABJ}}(N ; M)$.

There are various ways to analyze the ABJ partition function (3.1), including the Fermi gas approach [73, 104, 105] extensively used in the literature. However, for the purpose of studying its HS limit, the most convenient starting point is the "mirror description" of the ABJ partition function found in [92], generalizing the mirror description of the ABJM partition function $[106,107]$. The "mirror description" of the ABJ partition function is as follows:

$$
Z_{\mathrm{ABJ}}(N ; M)_{k}=i^{-N(N+M-1)} 2^{-N} k^{-N} q^{\frac{1}{6} M\left(M^{2}-1\right)} Z_{\mathrm{CS}}(M)_{k} \Psi(N ; M)_{k},
$$

where

$$
Z_{\mathrm{CS}}(M)_{k}=q^{-\frac{1}{12} M\left(M^{2}-1\right)} k^{-\frac{M}{2}} \prod_{j=1}^{M-1}\left(2 \sin \frac{\pi j}{k}\right)^{M-j}
$$

is the partition function for the $\mathrm{U}(M)_{k}$ CS theory and we defined the quantity ${ }^{10}$

$$
\Psi(N ; M)_{k} \equiv \frac{(-1)^{\frac{1}{2} N(N-1)}}{N_{1} !} \prod_{j=1}^{N}\left[\frac{-1}{2 \pi i} \int_{C} \frac{\pi d s_{j}}{\sin \left(\pi s_{j}\right)}\right] \prod_{j=1}^{N} \frac{\left(q^{s_{j}+1}\right)_{M}}{\left(-q^{s_{j}+1}\right)_{M}} \prod_{1 \leq j<m \leq N} \frac{\left(1-q^{s_{m}-s_{j}}\right)^{2}}{\left(1+q^{s_{m}-s_{j}}\right)^{2}} .
$$

In the above, we defined

$$
q \equiv e^{-\frac{2 \pi i}{k}}
$$

and $(a)_{n}=(a ; q)_{n} \equiv \prod_{j=0}^{n-1}\left(1-a q^{j}\right)$ is the $q$-Pochhammer symbol. The contour of integration in (3.9) is $C=[-i \infty+\eta,+i \infty+\eta]$ with the constant $\eta$ chosen to lie in the following range:

$$
\begin{cases}-M-1<\eta<0 & (k \geq 2 M) \\ -\frac{k}{2}-1<\eta<-\frac{k}{2}-M & (M \leq k \leq 2 M) .\end{cases}
$$

In [92], various consistency checks of the expression (3.7) were performed: (i) agreement of the perturbative expansion with the original matrix integral (3.1), (ii) vanishing of the partition function for $k<M$, in accord with the prediction [55] that there must be no SCFT in this range, and (iii) invariance under the Giveon-Kutasov-Seiberg duality (2.6). Later, the expression (3.7) was derived in [93] directly from the matrix integral (3.1) using the Cauchy-Vandermonde formula.

\footnotetext{
${ }^{10}$ Note that $\Psi$ defined in (3.9) is different from the one in [92] by the inclusion of the factor $(-1)^{\frac{1}{2} N(N-1)}$.
} 


\subsection{The large $M$ expansion}

We would like to develop a formulation to evaluate the ABJ partition function in the HS limit (1.1). The expression (3.7) is especially suitable for that purpose, since the number of integrals $N$ is fixed in the HS limit. To begin with, let us rewrite (3.9) in the following way [105]:

$$
\Psi(N ; M)_{k}=\frac{1}{N !}\left[\prod_{j=1}^{N} \int_{-\infty}^{\infty} d x_{j}\right] e^{\sum_{j=1}^{N} f\left(x_{j}\right)} \prod_{j<m}^{N} \tanh ^{2} \frac{\pi\left(x_{j}-x_{m}\right)}{k},
$$

where we did the following change of variables

$$
s_{j}=-\frac{M+1}{2}+i x_{j}, \quad j=1, \ldots, N,
$$

and also defined

$$
f(x, k, t)=\sum_{m=-\frac{M-1}{2}}^{\frac{M-1}{2}} \ln \tanh \frac{\pi(x+i m)}{k}-R(x),
$$

with

$$
R(x)= \begin{cases}\ln (2 \cosh (\pi x)) & (M=2 p: \text { even }), \\ \ln (2 \sinh (\pi x)) & (M=2 p-1: \text { odd }) .\end{cases}
$$

In (3.14), the summation over $m$ is done in steps of one; namely, $m=-\frac{M-1}{2},-\frac{M-1}{2}+$ $1, \ldots, \frac{M-1}{2}-1, \frac{M-1}{2}$, whether $M$ is even or odd. It is easy to show that the integration contour for $x_{j}$ in (3.12) corresponds to choosing $\eta$ correctly in the range (3.11), and that $x=0$ is the critical point of the function $f(x)$ for both even and odd $M$. Therefore, the strategy is to expand $f(x)$ around $x=0$ and carry out the integration by expansion around that point, taking into account the HS limit (1.1). It is easy to show that $f(x, k, t)$ is an even function in $x$.

As we have shown in appendix A, using the Euler-Maclaurin formula, $f(x, k, t)$ can be formally rewritten as

$$
f(x, k, t)=\frac{\cos \frac{2 x \partial_{t}}{k}}{\sinh \frac{\partial_{t}}{k}} \ln \tan \frac{\pi t}{2},
$$

in the sense that the formal power expansion of (3.16) around $x=0$ reproduces the formal power expansion of (3.14). Namely, the right hand side gives the asymptotic expansion of $f(x, k, t)$. Let us write the expansion of $(3.16)$ in $x$ as

$$
f(x, k, t) \equiv \sum_{n=0}^{\infty} \frac{(-1)^{n} f_{2 n}(k, t)}{(2 n) !} \frac{x^{2 n}}{k^{2 n-1}} .
$$

Here, the quantities $f_{2 n}(k, t)$ are defined as the expansion coefficients and their explicit expression is given by (3.16) as

$$
f_{2 n}(k, t)=k^{2 n-1} \frac{\left(\frac{2 \partial_{t}}{k}\right)^{2 n}}{\sinh \frac{\partial_{t}}{k}} \ln \tan \frac{\pi t}{2}=\sum_{m=0}^{\infty} \frac{2^{2 n}\left(2-2^{2 m}\right) B_{2 m}}{(2 m) ! k^{2 m}} \partial_{t}^{2 n+2 m-1} \ln \tan \frac{\pi t}{2},
$$


where $B_{n}$ are the Bernoulli numbers. Note that $f_{2 n}(k, t)$ is defined so that its $1 / k$ expansion (which is equivalent to the $1 / M$ expansion) starts with an $\mathcal{O}\left(k^{0}\right)$ term. The $m=0$ term in $f_{0}$ is understood as

$$
\frac{1}{\partial_{t}} \ln \tan \frac{\pi t}{2}=\int_{0}^{t} d y \ln \tan \frac{\pi y}{2}=-\frac{2}{\pi} \mathcal{I}\left(\frac{\pi t}{2}\right)
$$

where $\mathcal{I}(x)$ was defined in $(2.5)$.

If we write down the first few terms of the expansion (3.17), we have

$$
f(x, k, t)=k f_{0}(k, t)-\frac{f_{2}(k, t)}{2 !} \frac{x^{2}}{k}+\frac{f_{4}(k, t)}{4 !} \frac{x^{4}}{k^{3}}-\cdots
$$

The first term gives a constant contribution irrelevant for the $x$ integration, while the $x^{2}$ term suggests that we define a new variable $\xi$ by

$$
x=k^{1 / 2} \xi,
$$

so that the expansion (3.20) now reads

$$
f(x, k, t)=\sum_{n=0}^{\infty} \frac{(-1)^{n} f_{2 n}(k, t)}{(2 n) !} \frac{\xi^{2 n}}{k^{n-1}}=k f_{0}(k, t)-\frac{f_{2}(k, t)}{2 !} \xi^{2}+\frac{f_{4}(k, t)}{4 !} \frac{\xi^{4}}{k}+\cdots
$$

Now, the $\xi^{2}$ term is $\mathcal{O}\left(k^{0}\right)$ and the higher power terms in $\xi$ are down by powers of $1 / k$. This gives a starting point for the large $k$ (large $M$ ) expansion of the integral (3.12).

In terms of $\xi$, the integral (3.12) can be rewritten as

$$
\begin{aligned}
\Psi(N ; M)_{k}= & \frac{\pi^{N(N-1)} e^{k N f_{0}(k, t)}}{N ! k^{\frac{N^{2}}{2}}-N}\left[\prod_{j=1}^{N} \int_{-\infty}^{\infty} d \xi_{j}\right] \Delta(\xi)^{2} \\
& \times \exp \left[\sum_{n=1}^{\infty} \frac{(-1)^{n} f_{2 n}(k, t)}{(2 n) ! k^{n-1}} \sum_{j=1}^{N} \xi_{j}^{2 n}+2 \sum_{1 \leq j<m \leq N} \ln \frac{\tanh \frac{\pi\left(\xi_{j}-\xi_{m}\right)}{k^{1 / 2}}}{\frac{\pi\left(\xi_{j}-\xi_{m}\right)}{k^{1 / 2}}}\right]
\end{aligned}
$$

where $\Delta(\xi)$ is the Vandermonde determinant,

$$
\Delta(\xi) \equiv \prod_{1 \leq j<m \leq N}\left(\xi_{j}-\xi_{m}\right)
$$

The integral (3.23) is a standard Hermitian matrix integral and can be straightforwardly evaluated, regarding the $\xi^{2}$ term as giving the propagator and all higher power terms as interactions. Here we do not present the detail of the computation but simply write down 
the resulting large $M$ expansion:

$$
\begin{aligned}
\mathcal{F}(N ; M)_{k} \equiv-\ln \Psi(N ; M)_{k} \\
=\frac{2 N M}{\pi t} \mathcal{I}\left(\frac{\pi t}{2}\right)+\frac{N^{2}}{2} \ln \frac{4 M}{\pi t \sin (\pi t)}-\frac{N}{2} \ln \frac{2 M^{2}}{\pi t^{2}}-\ln G_{2}(N+1) \\
-\frac{N\left(2 N^{2}-1\right)}{48}\left(\frac{\pi t}{M \sin (\pi t)}\right)[3 \cos (2 \pi t)+1] \\
-\frac{N^{2}}{2304}\left(\frac{\pi t}{M \sin (\pi t)}\right)^{2}\left[\left(17 N^{2}+1\right) \cos (4 \pi t)+4\left(11 N^{2}-29\right) \cos (2 \pi t)-157 N^{2}+211\right] \\
-\frac{N}{552960}\left(\frac{\pi t}{M \sin (\pi t)}\right)^{3}\left[\left(674 N^{4}+250 N^{2}+201\right) \cos (6 \pi t)\right. \\
\quad-6\left(442 N^{4}+690 N^{2}-427\right) \cos (4 \pi t)+3\left(2282 N^{4}+3490 N^{2}-3635\right) \cos (2 \pi t) \\
\left.\quad+4348 N^{4}-21940 N^{2}+12750\right] \\
-\frac{N^{2}}{22118400}\left(\frac{\pi t}{M \sin (\pi t)}\right)^{4}\left[\left(6223 N^{4}+8330 N^{2}+2997\right) \cos (8 \pi t)\right. \\
\quad-8\left(3983 N^{4}+6730 N^{2}-363\right) \cos (6 \pi t)+20\left(3797 N^{4}+1870 N^{2}+1623\right) \cos (4 \pi t) \\
\left.\quad-8\left(22249 N^{4}-44410 N^{2}+37011\right) \cos (2 \pi t)-56627 N^{4}+113630 N^{2}-18753\right] \\
+\mathcal{O}\left(M^{-5}\right) .
\end{aligned}
$$

Note that the full ABJ free energy $F_{\mathrm{ABJ}}=-\ln Z_{\mathrm{ABJ}}$ contains more terms coming from (3.7). The computational detail of (3.25) can be found in appendix B. Because we used an asymptotic expansion in evaluating the integral, the large $M$ expansion (3.25) is also an asymptotic expansion to be completed by non-perturbative corrections.

As the last and important remark in this section, we emphasize that as is evident in (3.25), the $1 / M$ expansion organizes itself into the $G_{\mathrm{HS}}$ expansion, which lead us to the proposal in (2.3).

\section{The bulk side: $\mathcal{N}=6$ Vasiliev theory}

In this section we compute the one-loop free energy of the bulk HS theory dual to the ABJ theory in the higher spin limit (1.1). ${ }^{11}$ It was conjectured in [54] that the ABJ theory in the higher spin limit corresponds to the $\mathcal{N}=6$ parity-violating $\mathrm{U}(N)$ Vasiliev theory on $\mathrm{AdS}_{4}$. The Vasiliev theory has three parameters:

1. The Newton constant $G_{\mathrm{HS}}$ which is proportional to $M^{-1}$ at large $M$, as mentioned in the Introduction and section 2.

2. The rank $N$ of the $\mathrm{U}(N)$ Chan-Paton factors which is identified with the $N$ of the $\mathrm{U}(N) \times \mathrm{U}(N+M)$ gauge group of the ABJ theory.

3. The PV phase $\theta_{0}$ which violates parity and higher spin symmetry. As stated in the Introduction, $\theta_{0}$ is identified with the 't Hooft coupling $t$ by $\theta_{0}=\pi t / 2$ [54, 95].

\footnotetext{
${ }^{11}$ We thank Rajesh Gopakumar for stimulating discussions which motivated us to carry out the calculation in this section.
} 
The partition function of the Vasiliev theory takes the following form in perturbation theory:

$$
Z_{\mathrm{HS}} \equiv e^{-F_{\mathrm{HS}}} \quad \text { where } \quad F_{\mathrm{HS}}=\frac{1}{G_{\mathrm{HS}}} F_{\mathrm{HS}}^{(-1)}+F_{\mathrm{HS}}^{(0)}+G_{\mathrm{HS}} F_{\mathrm{HS}}^{(1)}+\cdots
$$

The free energy $F_{\mathrm{HS}}^{(\ell)}$ at $(\ell+1)$-loops is a function of the PV phase $\theta_{0}$ and may receive logarithmic corrections of the form $G_{\mathrm{HS}}^{\ell} \ln G_{\mathrm{HS}}$. The tree-level free energy $G_{\mathrm{HS}}^{-1} F_{\mathrm{HS}}^{(-1)}$ is the saddle point action of the Vasiliev theory. Although there are some propositions on the actions of the Vasiliev theory [96-99], it is not obvious to compute the tree level free energy from these actions. Thus we focus on the leading correction $F_{\mathrm{HS}}^{(0)}$, the one-loop free energy of the Vasiliev theory. The spectrum does not depend on the PV phase $\theta_{0}$, and we can compute $F_{\mathrm{HS}}^{(0)}$ in the standard manner [17-20, 33-36, 108].

\subsection{The one-loop contribution}

The $\mathcal{N}=6$ Vasiliev theory is constructed from the so-called $n=6$ extended supersymmetric Vasiliev theory by imposing a set of $\mathrm{SO}(6)$ invariant boundary conditions $[54,109]$. The parity-even $n=6$ Vasiliev theory can have 64 supercharges, but the boundary conditions and the parity violation reduce the number of supersymmetries to $\mathcal{N}=6$ with 24 supercharges. The spectrum of the $\mathcal{N}=6$ Vasiliev theory is given by $[54,109]$

- 32 fields for each integer, $s=0,1, \cdots$, and half-integer spin, $s=\frac{1}{2}, \frac{3}{2}, \frac{5}{2}, \ldots$ and their associated ghosts with spin $s-1$.

- All integer and half-integer spin fields with $s \geq 2$ obey the so-called $\Delta_{+}=s+1$ boundary condition at the $\mathrm{AdS}_{4}$ boundary, and their associated ghosts have $\Delta_{+}=$ $s+2$.

- Half of the spin-0 fields have the $\Delta_{+}=1$ boundary condition, whereas the other half $\Delta_{-}=2$.

- Except for one out of thirty-two, the $\mathrm{U}(N)$ spin-1 fields have the $\Delta_{+}=2$ boundary condition and $\Delta_{+}=3$ for the associated ghosts. The remaining one has the mixed boundary condition, $i \epsilon_{i j k}\left(\partial_{j} A_{k}+A_{j} A_{k}\right)+\tan (\pi t) \partial_{z} A_{i}=0$, with the boundary ChernSimons term at level $k$, corresponding to the gauging of the $\mathrm{U}(N)$ symmetry $[18,54]$.

- The spin-0 ghost field for the spin-1 field with the mixed boundary condition has the $\Delta_{-}=0$ boundary condition [18].

As our strategy, to avoid the technical difficulty caused by the mixed boundary condition, we only deal with the regime $t \ll 1$ where the spin- 1 field with the mixed boundary condition in effect has $\Delta=2+\mathcal{O}(t) \simeq 2$ and then infer the form of the one-loop free energy for generic $t$ from this data in conjunction with the result of [95]. 
- Spin-s fields

\begin{tabular}{|c|c|c|c|c|c|c|}
\hline spin & 0 & 0 & 1 & 1 (gauge) & $s \geq 2$ & $s=\mathbb{Z}_{\geq 0}+\frac{1}{2}$ \\
\hline no. of fields & 16 & 16 & 31 & 1 & 32 & 32 \\
\hline boundary cond. & $\Delta_{+}=1$ & $\Delta_{-}=2$ & $\Delta_{+}=2$ & $\Delta \simeq 2$ (mixed) & $\Delta_{+}=s+1$ & $\Delta_{+}=s+1$ \\
\hline
\end{tabular}

- Spin- $(s-1)$ ghosts

\begin{tabular}{|c|c|c|c|c|c|c|}
\hline spin & N/A & N/A & 0 & 0 (gauge) & $s-1 \geq 1$ & $s-1=\mathbb{Z}_{\geq 0}+\frac{1}{2}$ \\
\hline no. of fields & N/A & N/A & 31 & 1 & 32 & 32 \\
\hline boundary cond. & N/A & N/A & $\Delta_{+}=3$ & $\Delta_{-}=0+\frac{c_{-}\left(\theta_{0}\right)}{M}$ & $\Delta_{+}=s+2$ & $\Delta_{+}=s+2$ \\
\hline
\end{tabular}

Table 1. The spectrum of the $\mathcal{N}=6$ Vasiliev theory (in the regime $t \ll 1$ ) labeled by spin, number of fields, and boundary conditions and associated ghosts. Note, in particular, the $\mathcal{O}(1 / M)$ correction to the $\Delta_{-}$spin-0 ghost for the spin- 1 gauge field, where $c_{-}\left(\theta_{0}\right)$ is known up to a numerical constant. The dimension of other fields also receives $\mathcal{O}(1 / M)$ corrections which, however, do not contribute to the one-loop free energy. As mentioned above, the spin 1 field with the mixed boundary condition has $\Delta=2+\mathcal{O}(t) \simeq 2$ in the regime $t \ll 1$.

We summarize the spectrum in table 1 . There is a very important point to be stressed: the boundary conditions, as stated here, are only true in the strict large $M$ limit. In fact, $\Delta_{ \pm}$are the dimensions of CFT operators dual to higher spin fields and may thus receive $1 / M$ corrections which moreover depend on the PV phase $\theta_{0}[95,110]$. As we will see, the $1 / M$ correction to the $\Delta_{-}$spin- 0 ghost fields are particularly important and contribute to the one-loop free energy, whereas all the rest of $1 / M$ corrections, even if present, have no contributions to one-loop. In table 1 we indicated the $\mathcal{O}(1 / M)$ correction to the $\Delta_{-}$spin- 0 ghost to emphasize this point.

We can now write down the bulk one-loop partition function. Taking into account the $\mathrm{U}(N)$ Chan-Paton factors, it reads

$$
e^{-F_{\mathrm{HS}}^{(0)}}=\left[Z_{0, \Delta_{+}}^{16} Z_{0, \Delta_{-}}^{16} Z_{1, \Delta_{+}}^{31} Z_{1, \Delta} \prod_{s=2}^{\infty} Z_{s, \Delta_{+}}^{32} \prod_{s=0}^{\infty} Z_{s+\frac{1}{2}, \Delta_{+}}^{32}\right]^{N^{2}}
$$

where $Z_{s, \Delta_{ \pm}}$is the partition function for a field with spin $s$ and the boundary condition $\Delta_{ \pm}$ and can be expressed in terms of functional determinants of symmetric transverse traceless (STT) tensors in $\mathrm{AdS}_{4}[17,19,20,108]:^{12}$

$$
Z_{s, \Delta_{ \pm}}=\left\{\begin{array}{ll}
{\left[\frac{\operatorname{det}_{s-1, \Delta_{ \pm}}^{\mathrm{STT}}\left[-\nabla^{2}+(s+1)(s-1)\right]}{\operatorname{det}_{s, \Delta_{ \pm}}^{\mathrm{STT}}\left[-\nabla^{2}+(s+1)(s-2)-s\right]}\right]^{1 / 2}} & \text { for } s \in \mathbb{Z}_{\geq 0} \\
{\left[\frac{\operatorname{det}_{s, \Delta_{ \pm}}^{\mathrm{STT}}\left[-\not^{2}+(s-1 / 2)^{2}\right]}{\operatorname{det}_{s-1, \Delta_{ \pm}}^{\mathrm{STT}}\left[-\not^{2}+(s+1 / 2)^{2}\right]}\right]^{1 / 4}} & \text { for } s \in \mathbb{Z}_{\geq 0}+\frac{1}{2}
\end{array},\right.
$$

\footnotetext{
${ }^{12}$ In the unit $R_{\mathrm{AdS}}=1$.
} 
with the understanding that

$$
\operatorname{det}_{s}^{\mathrm{STT}}[\cdots]=1 \quad \text { for } \quad s<0 .
$$

$Z_{1, \Delta}$ is the partition function for the spin-1 gauge field with the mixed boundary condition in the regime $t \ll 1$, corresponding effectively to $\Delta \simeq \Delta_{+}=2$, and its associated ghost with the $\Delta_{-}$boundary condition, and a similar one-loop determinant formula holds for $Z_{1, \Delta}$. The spin- $(s-1)$ determinants in (4.3) are the contributions from the gauge fixing ghosts. These determinants can be explicitly computed by applying the techniques developed in [111-113]. To proceed, we first simplify (4.2) by using the result of Giombi and Klebanov for the type-A Vasiliev theory [17],

$$
Z_{\text {type A }}=\prod_{s=0}^{\infty} Z_{s, \Delta_{+}}=1
$$

Dividing (4.2) by $\left(Z_{\text {type A }}\right)^{32 N^{2}}$ yields

$$
e^{-F_{\mathrm{HS}}^{(0)}}=\left[\left(\frac{Z_{0, \Delta_{-}}}{Z_{0, \Delta_{+}}}\right)^{16} \frac{Z_{1, \Delta}}{Z_{1, \Delta_{+}}} \prod_{s \in \mathbb{Z}_{\geq 0}+\frac{1}{2}} Z_{s, \Delta_{+}}^{32}\right]^{N^{2}} .
$$

Thus the bosonic contribution to the one-loop free energy could come only from the spin-0 and spin-1 fields. This simplifies the calculation.

For the convenience of the subsequent calculations we introduce

$$
F_{(\Delta, s)}= \begin{cases}\frac{1}{2} \ln \operatorname{det}_{s}^{\mathrm{STT}}\left[-\nabla^{2}+\left(\Delta-\frac{3}{2}\right)^{2}-s-\frac{9}{4}\right] & \text { for } s \in \mathbb{Z} \\ \frac{1}{2} \ln \operatorname{det}_{s}^{\mathrm{STT}}\left[-\not^{2}+\left(\Delta-\frac{3}{2}\right)^{2}\right] & \text { for } s \in \mathbb{Z}+\frac{1}{2}\end{cases}
$$

which has been computed by Camporesi and Higuchi [111-113] and is given in terms of the spectral zeta function

$$
F_{(\Delta, s)}=-\frac{1}{2} \zeta_{(\Delta, s)}^{\prime}(0)-\frac{1}{2} \zeta_{(\Delta, s)}(0) \ln \left(\Lambda^{2}\right),
$$

where the spectral zeta function $\zeta_{(\Delta, s)}(z)$ is defined by

$$
\begin{aligned}
\zeta_{(\Delta, s)}(z) & =\frac{8(2 s+1)}{3 \pi} \int_{0}^{\infty} d u \frac{\mu_{s}(u)}{\left[u^{2}+(\Delta-3 / 2)^{2}\right]^{z}}, \quad \zeta_{(\Delta, s)}^{\prime}(z)=\frac{\partial}{\partial z} \zeta_{(\Delta, s)}(z), \\
\mu_{s}(u) & =\frac{\pi u}{16}\left[u^{2}+\left(s+\frac{1}{2}\right)^{2}\right] \tanh (\pi(u+i s)) .
\end{aligned}
$$

The parameter $\Lambda$ in (4.8) is a UV cutoff. The logarithmic divergence arises in even dimensions and is related to the conformal anomaly. As we will show below, the logarithmic divergence actually cancels out in the $\mathcal{N}=6$ Vasiliev theory (in a certain regularization scheme). Hence the net contribution to the one-loop partition function comes solely from $\zeta_{(\Delta, s)}^{\prime}$. In particular, the $\mathcal{O}(\ln M)$ correction observed in the ABJ theory comes entirely from the $\Delta_{-}$spin-0 ghosts for the spin-1 $\mathrm{U}(N)$ gauge fields and the consequence of the "induced gauge symmetry" [18]. 


\subsection{The bosonic contributions}

We first consider the bosonic part $F_{\mathrm{HS}, \mathrm{B}}^{(0)}$ of the one-loop free energy. As commented on below (4.6), there are only contributions from the spin-0 and spin-1 fields. Moreover, as it will turn out, it is free of logarithmic divergences. For integer spins, the spectral zeta function $\zeta_{(\Delta, s)}(0)$ has been calculated by Camporesi and Higuchi [17, 111]:

$$
\zeta_{(\Delta, s)}(0)=\frac{2 s+1}{24}\left[\nu^{4}-\left(s+\frac{1}{2}\right)^{2}\left(2 \nu^{2}+\frac{1}{6}\right)-\frac{7}{240}\right] \quad \text { with } \quad \nu=\Delta-\frac{3}{2} .
$$

Noting that $\Delta_{+}-3 / 2=-\left(\Delta_{-}-3 / 2\right)$, this expression implies, due to the invariance under $\nu \rightarrow-\nu$, that

$$
\zeta_{\left(\Delta_{+}, s\right)}(0)=\zeta_{\left(\Delta_{-}, s\right)}(0)
$$

Thus the logarithmic divergence in the bosonic part of the free energy cancel out between the contributions from different boundary conditions, namely,

$$
\left.\ln \frac{Z_{0, \Delta_{-}}}{Z_{0, \Delta_{+}}}\right|_{\log \text { div }}=0,\left.\quad \ln \frac{Z_{1, \Delta}}{Z_{1, \Delta_{+}}}\right|_{\log \text { div }}=0,
$$

where $\left.\ldots\right|_{\log \text { div }}$ means the logarithmically divergent part read off from (4.8).

Turning to the finite piece, we first calculate the spin-1 free energy. Again borrowing the result from $[17,111]$ and paying special attention to the ghost boundary conditions, we have ${ }^{13}$

$$
\ln \frac{Z_{1, \Delta}}{Z_{1, \Delta_{+}}}=\frac{1}{2}\left(I_{B}\left(\Delta_{+}-3 / 2,0\right)-I_{B}\left(\Delta_{-}-3 / 2,0\right)\right),
$$

where

$$
I_{B}(\nu, s)=\frac{2 s+1}{3} \int_{0}^{\nu} d x\left[\left(s+\frac{1}{2}\right)^{2} x-x^{3}\right] \psi(x+1 / 2)
$$

with $\psi(z)$ being the digamma function. Here, as emphasized in the discussion of the spectrum, we need special care in dealing with the conformal dimensions $\Delta_{ \pm}$. Generically, the dimensions $\Delta_{ \pm}$may receive the finite $M$ corrections, and for the spin- 0 ghosts it reads

$$
\Delta_{+}=3+\frac{c_{+}\left(\theta_{0}\right)}{M}+\mathcal{O}\left(\frac{1}{M^{2}}\right), \quad \Delta_{-}=0+\frac{c_{-}\left(\theta_{0}\right)}{M}+\mathcal{O}\left(\frac{1}{M^{2}}\right),
$$

where $c_{ \pm}\left(\theta_{0}\right)$ are functions of the PV phase $\theta_{0}$. In fact, it has been shown $[95,110]$ that the $\mathcal{O}(1 / M)$ corrections exist in three-dimensional interacting CFTs with pseudo-higher spin symmetries. When we take into account the $\mathcal{O}(1 / M)$ corrections, an explicit calculation shows that

$$
I_{B}\left(\Delta_{+}-3 / 2,0\right)=\mathcal{O}\left(M^{0}\right), \quad I_{B}\left(\Delta_{-}-3 / 2,0\right)=+\ln \left(M / c_{-}\left(\theta_{0}\right)\right)+\mathcal{O}\left(M^{0}\right),
$$

where the $\mathcal{O}\left(M^{0}\right)$ terms are independent of $c_{ \pm}\left(\theta_{0}\right)$. We thus find that

$$
\ln \frac{Z_{1, \Delta}}{Z_{1, \Delta_{+}}}=-\frac{1}{2} \ln \left(M / c_{-}\left(\theta_{0}\right)\right)+\mathcal{O}\left(M^{0}\right) .
$$

\footnotetext{
${ }^{13}$ To be more precise, there is a contribution from the spin 1 fields, $\frac{1}{2}\left(I_{B}(\Delta-3 / 2,1)-I_{B}\left(\Delta_{+}-3 / 2,1\right)\right)$, which, however, is at most of order $\mathcal{O}(t)$ and negligible for our purpose.
} 
Since there is an unknown numerical constant in $c_{-}\left(\theta_{0}\right)$, we cannot accurately calculate the $\mathcal{O}\left(M^{0}\right)$ term. Similarly, it is straightforward to find the spin-0 free energy as

$$
\ln \frac{Z_{0, \Delta_{-}}}{Z_{0, \Delta_{+}}}=\frac{1}{2}\left(-I_{B}(-1 / 2,0)+I_{B}(1 / 2,0)\right)=\mathcal{O}\left(M^{0}\right) .
$$

Combining (4.17) and (4.18) together, we conclude that the bosonic part of the bulk oneloop free energy is

$$
F_{\mathrm{HS}, \mathrm{B}}^{(0)}=+\frac{N^{2}}{2} \ln \left(M / c_{-}\left(\theta_{0}\right)\right)+\mathcal{O}\left(M^{0}\right) .
$$

We will later discuss the form of $c_{-}\left(\theta_{0}\right)$ in section 5 .

\subsection{The fermionic contributions}

We next consider the fermionic part $F_{\mathrm{HS}, \mathrm{F}}^{(0)}$ of the one-loop free energy. Again, as it will turn out, it is free of logarithmic divergences. Moreover, it has no $\ln M$ corrections.

We first show the absence of the logarithmic divergences: for $s \in \mathbb{Z}+1 / 2$, we can rewrite the spectral zeta function $\zeta_{(\Delta, s)}(z)$ as a sum of two terms

where

$$
\zeta_{(\Delta, s)}(z)=\frac{8(2 s+1)}{3 \pi}\left(g_{1}(\nu, s ; z)+g_{2}(\nu, s ; z)\right)
$$

$$
\begin{aligned}
& g_{1}(\nu, s ; z)=\frac{\pi}{16} \int_{0}^{\infty} d u \frac{u}{\left(u^{2}+\nu^{2}\right)^{z}}\left[u^{2}+\left(s+\frac{1}{2}\right)^{2}\right] \\
& g_{2}(\nu, s ; z)=\frac{\pi}{8} \int_{0}^{\infty} d u \frac{u}{\left(u^{2}+\nu^{2}\right)^{z}\left(e^{2 \pi u}-1\right)}\left[u^{2}+\left(s+\frac{1}{2}\right)^{2}\right] .
\end{aligned}
$$

By explicit calculations, these two terms are given by

$$
g_{1}(\nu, s ; 0)=\frac{\pi \nu^{2}}{64}\left[\nu^{2}-\left(s+\frac{1}{2}\right)^{2}\right], \quad g_{2}(\nu, s ; 0)=\frac{\pi(20 s(s+1)+7)}{3840} .
$$

Meanwhile, from (4.6) and (4.8), the logarithmically divergent piece of $F_{\mathrm{HS}, \mathrm{F}}^{(0)}$ is

$$
-8 N^{2}\left[\zeta_{(3 / 2,1 / 2)}(0)+\sum_{s \in \mathbb{Z}_{\geq 0}+1 / 2}\left(\zeta_{(s+1, s)}(0)-\zeta_{(s+2, s-1)}(0)\right)\right] \ln \left(\Lambda^{2}\right) .
$$

This sum, as it stands, is divergent, and must be regularized. We adopt the regularization used in the analysis [20]. ${ }^{14}$ This yields

$$
\begin{aligned}
\left.F_{\mathrm{HS}, \mathrm{F}}^{(0)}\right|_{\log \text { div }} & =-8 N^{2}\left[\zeta_{(3 / 2,1 / 2)}(0)+\lim _{\alpha \rightarrow 0} \sum_{s \in \mathbb{Z}_{\geq 0}+1 / 2} s^{-\alpha}\left(\zeta_{(s+1, s)}(0)-\zeta_{(s+2, s-1)}(0)\right)\right] \ln \left(\Lambda^{2}\right) \\
& =32\left[\frac{11}{360}+\lim _{\alpha \rightarrow 0} \sum_{s \in \mathbb{Z}_{\geq 0}+1 / 2} s^{-\alpha}\left(-\frac{5 s^{4}}{12}+\frac{5 s^{2}}{24}+\frac{13}{2880}\right)\right] \ln \left(\Lambda^{2}\right)=0, \quad
\end{aligned}
$$

\footnotetext{
${ }^{14}$ This regularization can be slightly generalized to:

$$
\zeta_{(3 / 2,1 / 2)}(0)+\lim _{\alpha \rightarrow 0} \sum_{s \in \mathbb{Z}_{\geq 0}+1 / 2}(s+x)^{-\alpha} \zeta_{(s+1, s)}(0)-\lim _{\alpha \rightarrow 0} \sum_{s \in \mathbb{Z}_{\geq 0}+1 / 2}(s+y)^{-\alpha} \zeta_{(s+2, s-1)}(0) .
$$
}

One can show that this vanishes so long as $x+y=0$. 
where we used (4.22) to find the second line. Thus the fermionic part of the one-loop free energy is also free of logarithmic divergences.

We next evaluate the finite part. For $s \in \mathbb{Z}_{\geq 0}+1 / 2$, an explicit computation yields

$$
\begin{aligned}
\zeta_{(\Delta, s)}^{\prime}(0)= & -\frac{8(2 s+1)}{3 \pi}\left((s+1 / 2)^{2} d_{1}+d_{3}\right)+I_{F}(\nu, s) \\
& -\frac{(2 s+1)}{72} \nu\left(-3 \nu^{3}+4 \nu^{2}+\nu-12 s^{2}-12 s-3\right),
\end{aligned}
$$

where

$$
d_{n}=\frac{\pi}{8} \int_{0}^{\infty} d u \frac{u^{n} \ln u^{2}}{e^{2 \pi u}-1}, \quad I_{F}(\nu, s)=\frac{2 s+1}{3} \int_{0}^{\nu} d x\left[\left(s+\frac{1}{2}\right)^{2} x-x^{3}\right] \psi(x) .
$$

It is then straightforward to show that each piece in the finite part is of order $\mathcal{O}\left(M^{0}\right)$,

$$
\zeta_{(s+1, s)}^{\prime}(0)=\mathcal{O}\left(M^{0}\right), \quad \zeta_{(s+2, s-1)}^{\prime}(0)=\mathcal{O}\left(M^{0}\right)
$$

Hence the $\mathcal{O}(\ln M)$ contribution is absent in the fermionic free energy, and it is at most of order $\mathcal{O}\left(M^{0}\right)$,

$$
F_{\mathrm{HS}, \mathrm{F}}^{(0)}=\mathcal{O}\left(M^{0}\right) \text {. }
$$

\subsection{The full one-loop free energy}

Altogether, we find the full bulk one-loop free energy to be

$$
F_{\mathrm{HS}}^{(0)}=F_{\mathrm{HS}, \mathrm{B}}^{(0)}+F_{\mathrm{HS}, \mathrm{F}}^{(0)}=+\frac{N^{2}}{2} \ln \left(M / c_{-}\left(\theta_{0}\right)\right)+\mathcal{O}\left(M^{0}\right) .
$$

Note that the leading $\mathcal{O}(\ln M)$ contribution comes entirely from the $\Delta_{-}$spin-0 ghosts for the spin-1 $\mathrm{U}(N)$ gauge fields and, as in [18], is the consequence of the "induced gauge symmetry."

The bulk one-loop free energy (4.30) is consistent with the $\mathcal{O}(\ln M)$ correction to the ABJ free energy with the identification (2.3) of the Newton constant

$$
G_{\mathrm{HS}}=\frac{\gamma}{M} \frac{\pi t}{\sin (\pi t)}
$$

We are, however, unable to determine the constant $\gamma$ which requires the precise value of the $\mathcal{O}\left(M^{0}\right)$ correction. ${ }^{15}$ We will make further comments on $c_{-}\left(\theta_{0}\right)$ in the one-loop free energy in the next section.

\footnotetext{
${ }^{15}$ Once again, as remarked in footnote 7 , the constant $\gamma$ has been recently determined to be $\gamma=2 / \pi$ by one of the authors in [100].
} 


\section{Discussions}

In the last two sections, we have calculated the free energies of the ABJ theory in the HS limit and the $\mathcal{N}=6$ Vasiliev theory at one-loop. We are now ready to discuss the correspondence between the two theories. However, it is not as straightforward as comparing the free energy of the ABJ theory (3.25) and that of the $\mathcal{N}=6$ HS theory (4.30) as they are, and it requires some considerations to make the correspondence more precise.

As already mentioned in section 2, the ABJ theory, even in the HS limit (1.1), has more degrees of freedom than necessary to describe the $\mathcal{N}=6$ HS dual. For instance, the free energy of the ABJ theory in the limit (1.1) goes as $M^{2}$, since the ABJ theory is a theory of $\mathrm{U}(M)$ matrices. On the other hand, the free energy of the HS theory is expected to grow as $M$, reflecting the fact that it is dual to a $\mathrm{U}(M)$ vector model. The $M^{2}$ growth comes from the $\mathrm{U}(M)$ part of the $\mathrm{U}(N) \times \mathrm{U}(N+M)$ CS free energy. In the case of $\mathrm{U}(M)$ CS theory coupled to fundamental matter [114], the $\mathcal{O}(M)$ growth was extracted by normalizing the CS partition function to be unity, or equivalently, dividing the full partition function by the CS partition function. In our case, however, the situation is more involved, since the gauge group is a product group $\mathrm{U}(N) \times \mathrm{U}(N+M)$ and the ABJ theory has bi-fundamental matter.

Here we first recall our proposal made in section 2 and then elaborate on it. The proposed correspondence is given in (2.2):

$$
\frac{Z_{\mathrm{HS}}\left(G_{\mathrm{HS}}, \theta_{0} ; N\right)}{\operatorname{Vol}(\mathrm{U}(N))}=Z_{\mathrm{vec}}(M ; N)_{k},
$$

where the "vector model subsector" of the partition function is identified as

$$
Z_{\mathrm{vec}}(M ; N)_{k}=\frac{\left|Z_{\mathrm{ABJ}}(N, N+M)_{k}\right|}{Z_{\mathrm{CS}}(M)_{k}} .
$$

In addition to the quotient by the $\mathrm{U}(M)$ CS partition function on the r.h.s., the l.h.s. of $(5.1)$ is divided by the $\mathrm{U}(N)$ volume, $\operatorname{Vol}(\mathrm{U}(N))=(2 \pi)^{\frac{N}{2}(N+1)} / G_{2}(N+1)$. This is the natural normalization for the bulk $\mathrm{U}(N)$ theory. The main idea behind (5.2) is to regard the open strings stretched between $N$ regular and $M$ fractional (and $N$ regular) D3-branes as the vector degrees of freedom dual to the HS theory, as illustrated in figure 1 for the type IIB brane construction of the ABJ(M) theory. Thus the quotient by $Z_{\mathrm{CS}}(M)_{k}$ is to remove contributions from the diagrams that only involve open strings whose both ends are on $M$ fractional D3-branes. As quantitative justifications, we note that the free energy $F_{\text {vec }}=-\ln Z_{\text {vec }}$ of the vector model subsector has the following properties:

1. $F_{\text {vec }}$ scales as $M \propto G_{\mathrm{HS}}^{-1}$ at the leading order in the HS limit (and of order $\mathcal{O}\left(N^{2}\right)$ when expressed in terms of the bulk 't Hooft coupling $\lambda_{\mathrm{HS}}=N G_{\mathrm{HS}}$, as it should be for $\mathrm{U}(N)$ theory).

2. $F_{\text {vec }}$ enjoys the Giveon-Kutasov-Seiberg duality (2.6), namely,

$$
F_{\text {vec }}(M ; N)_{k}=F_{\text {vec }}(|k|-M ; N)_{-k} .
$$

3. The leading logarithmic correction agrees with the bulk one-loop result (4.30),

$$
F_{\mathrm{vec}}(M ; N)_{k}=\cdots+\frac{N^{2}}{2} \ln M+\cdots .
$$


We have already emphasized the importance of the first property. Meanwhile, the second property might look a matter of aesthetics. However, the duality invariance (5.3) ensures the parity symmetry restoration at $\theta_{0}=0$ and $\frac{\pi}{2}$ with the identification $\theta_{0}=\pi t / 2$ where $t=M /|k|$, as required by the PV Vasiliev theory [54]. Had it been the $\mathrm{U}(N+M) \mathrm{CS}$ partition function $Z_{\mathrm{CS}}(N+M)_{k}$ to be divided in (5.2), the duality invariance would not have been respected. This vindicates the quotient by the $\mathrm{U}(M)$ CS partition function $Z_{\mathrm{CS}}(M)_{k}$ as opposed to $Z_{\mathrm{CS}}(N+M)_{k}$. Lastly, as already stated in previous sections, the third property implies the agreement between the ABJ and HS theories, provided that the HS Newton constant is identified as

$$
G_{\mathrm{HS}}=\frac{\gamma}{M} \frac{\pi t}{\sin (\pi t)} \stackrel{t \rightarrow 0}{\longrightarrow} \frac{\gamma}{M}
$$

which agrees with the one suggested in [101] for non-supersymmetric theories. We emphasize once again that the HS Newton constant $G_{\mathrm{HS}}$, rather than simply $1 / M$, is the expansion parameter that appears in the systematic $1 / M$ expansion (3.25) of the ABJ free energy. To this end, we spell out the free energy for the r.h.s. of (5.2) which lead to the main result (2.4):

$$
\begin{aligned}
F_{\mathrm{vec}}(M ; N)_{k}= & \operatorname{Re}\left[F_{\mathrm{ABJ}}(N, N+M)_{k}\right]-F_{\mathrm{CS}}(M)_{k} \\
= & \frac{2 N M}{\pi t} \mathcal{I}\left(\frac{\pi t}{2}\right)+\frac{N^{2}}{2} \ln \left(\frac{2}{\pi} \frac{M \sin (\pi t)}{\pi t}\right)-\frac{N^{2}}{2} \ln \left(\sin ^{2}(\pi t)\right) \\
& +\ln (\operatorname{Vol}(\mathrm{U}(N)))+\mathcal{O}(\pi t /(M \sin (\pi t))) .
\end{aligned}
$$

As promised, we would like to add more comments on the logarithmic terms in the second line. The first logarithmic term is identified with $+N^{2} / 2 \ln \left(G_{\mathrm{HS}}^{-1}\right)$ up to a numerical constant as in (2.4). As noted in the end of section 2, the second logarithmic term diverges as $t \rightarrow 0$ or 1 , and this might look like an unpleasant result. However, we now argue that this is indeed precisely the result predicted in [95] from HS symmetry considerations. ${ }^{16}$ To see it, note that comparing these two terms with the HS one-loop result (4.30), we wish to show that

$$
\frac{c_{-}\left(\theta_{0}\right)}{M}=G_{\mathrm{HS}} \sin ^{2}(\pi t)
$$

up to a numerical constant. In [95] it was suggested in eq. (A.5) that

$$
\frac{c_{-}\left(\theta_{0}\right)}{M}=a G_{\mathrm{HS}} \frac{\tilde{\lambda}^{2}}{1+\tilde{\lambda}^{2}}+b G_{\mathrm{HS}} \frac{\tilde{\lambda}^{2}}{\left(1+\tilde{\lambda}^{2}\right)^{2}}
$$

where $a$ and $b$ are unknown constants. Meanwhile, $\tilde{\lambda}$ for the $\mathcal{N}=6$ theory was conjectured in [54] to be

$$
\tilde{\lambda}=\tan \left(2 \theta_{0}\right)=\tan (\pi t) .
$$

\footnotetext{
${ }^{16}$ Two comments are in order: (1) Due to the $\mathrm{U}(N)$ symmetry and supersymmetries, the spectrum of the $\mathcal{N}=6$ theory is larger than that assumed in [95]. Thus, strictly speaking, we are pushing the applicability of their results potentially beyond the limits. (2) This argument of [95] applies to dimensions of CFT operators dual to higher spin fields. We are, however, applying their result to dimensions of bulk ghosts, even though there are no CFT operators dual to them. It is, however, reasonable to assume that the $O(1 / M)$ corrections to the dimensions of spin $(s-1)$-ghosts appear in the same form as those of their associated spin $s$-fields.
} 
Provided that $b=0$ for the $\mathcal{N}=6$ theory, it indeed yields

$$
\frac{c_{-}\left(\theta_{0}\right)}{M}=a G_{\mathrm{HS}} \sin ^{2}(\pi t)
$$

as we wished. It should also be noted that from the field theory viewpoint, the ABJ theory is related to the $\mathcal{N}=3 \mathrm{U}(N+M)_{-k}$ Chern-Simons-matter theory with $2 N$ fundamental hypermultiplets by gauging the $\mathrm{U}(N)$ subgroup of the flavour symmetry. The logarithmic singularity (as well as $+\frac{N^{2}}{2} \ln M$ term) is nothing but the one which appears in the difference of the free energies of the ABJ and the $\mathcal{N}=3$ theories and similar to the one in eq. (4.18) of [18].

We believe that all indicate our proposal (5.1) and (5.2) is at work. However, it is worth noting that the "vector model subsector" may be a misnomer, since open strings stretched between $M$ fractional and $N$ regular D3-branes, corresponding to the $\mathrm{U}(M)$ vector, do couple with open strings which ends only on $M$ fractional D3-branes, corresponding to the $\mathrm{U}(M)$ adjoint. Although the quotients (5.2) do remove all diagrams that only involve the latter degrees of freedom, it is not the case that these degrees of freedom do not appear at all in Feynman diagrams.

\section{Acknowledgments}

We thank Robert de Mello Koch, Yasuaki Hikida, Antal Jevicki, Rajesh Gopakumar, Shiraz Minwalla, Sanefumi Moriyama, Keita Nii, Eric Perlmutter, Mukund Rangamani, Joao Rodrigues, Xi Yin, and Costas Zoubos for useful discussions. SH would like to thank the Graduate School of Mathematics, Nagoya university, and the Yukawa Institute for Theoretical Physics for their hospitality at various stages of this work. The work of SH was supported in part by the National Research Foundation of South Africa and DSTNRF Centre of Excellence in Mathematical and Statistical Sciences (CoE-MaSS). Opinions expressed and conclusions arrived at are those of the author and are not necessarily to be attributed to the NRF or the CoE-MaSS. The work of KO was supported in part by JSPS Grant-in-Aid for Young Scientists (B) 23740178. MS is grateful to the Weizmann Institute for the stimulating environment at the "Black Holes and Quantum Information" workshop. The work of MS was supported in part by Grant-in-Aid for Young Scientists (B) 24740159 from the Japan Society for the Promotion of Science (JSPS).

\section{A Formal expansion of $f(x, k, t)$}

In this appendix, we derive the formal expansion (3.16) of the quantity $f(x, k, t)$ defined in (3.14).

First, let us do the following trivial rewriting of (3.14) as

$$
f(x, k, t)=\sum_{m=-\frac{M-1}{2}}^{\frac{M-1}{2}} \ln \frac{\tanh \frac{\pi(x+i m)}{k}}{\frac{\pi(x+i m)}{k}}+\sum_{m=-\frac{M-1}{2}}^{\frac{M-1}{2}} \ln \frac{\pi(x+i m)}{k}-R(x) .
$$


The quantity $f_{2 n}(k, t)$, which was defined in (3.17) and can be written as

$$
f_{2 n}(k, t)=\left.(-1)^{n} k^{2 n-1} \partial_{x}^{2 n} f(x, k, t)\right|_{x=0},
$$

is computed from the expression (A.1) as follows. First, for even $M,{ }^{17}$

$$
f_{2 n}= \begin{cases}k^{-1}\left[\sum_{m=-\frac{M-1}{2}}^{\frac{M-1}{2}} \ln \frac{\tan \frac{\pi m}{k}}{\frac{\pi m}{k}}+2 \sum_{m=\frac{1}{2}}^{\frac{M-1}{2}} \ln \frac{\pi m}{k}-\ln 2\right] & (n=0), \\ k^{2 n-1}\left[\sum_{m=-\frac{M-1}{2}}^{\frac{M-1}{2}} \partial_{m}^{2 n} \ln \frac{\tan \frac{\pi m}{k}}{\frac{\pi m}{k}}-2(2 n-1) ! \sum_{m=\frac{1}{2}}^{\frac{M-1}{2}} \frac{1}{m^{2 n}}-(-1)^{n} \frac{(2 \pi)^{2 n}\left(2^{2 n}-1\right) B_{2 n}}{2 n}\right] & (n \geq 1) .\end{cases}
$$

Here, we used the relation $\partial_{x}=-i \partial_{m}$ and the formula [115, eq. 1.518.2]

$$
R^{M: \text { even }}(x)=\ln (2 \cosh (\pi x))=\ln 2+\sum_{n=1}^{\infty} \frac{(2 \pi)^{2 n}\left(2^{2 n}-1\right) B_{2 n}}{2 n(2 n) !} x^{2 n} .
$$

For odd $M$, some care is needed in setting $x=0$, because the singularity at $x=0$ coming from the $m=0$ term in the second sum of (A.1) cancels against the singularity coming from $R(x)$. Using the formula [115, eq. 1.518.1]

$$
R^{M: \text { odd }}(x)=\ln (2 \sinh (\pi x))=\ln (2 \pi x)+\sum_{n=1}^{\infty} \frac{(2 \pi)^{2 n} B_{2 n}}{2 n(2 n) !} x^{2 n},
$$

we obtain, for odd $M$,

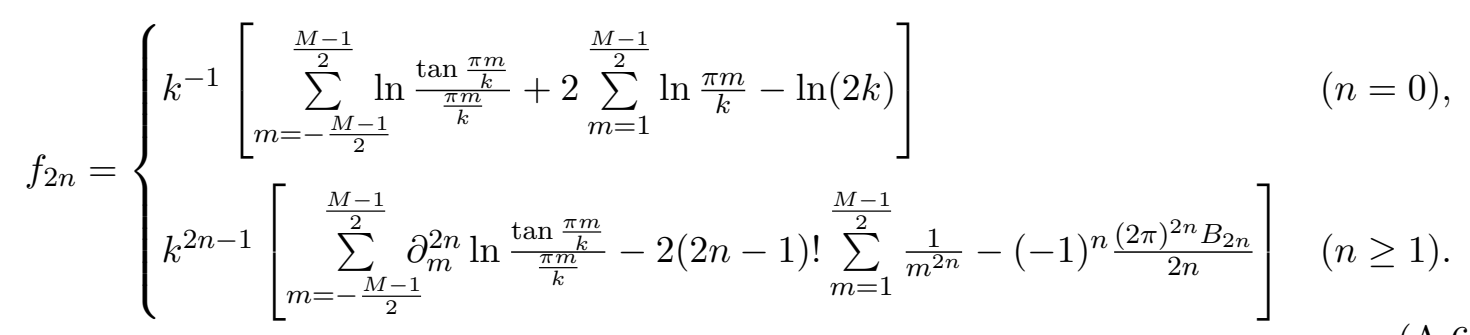

Because the summand in the first terms of (A.3), (A.6) is regular at $m=0$ thanks to the rewriting (A.1), it can be safely evaluated using the Euler-Maclaurin formula. The version of the Euler-Maclaurin formula relevant here is the one that uses the midpoint trapezoidal rule and is given by (see e.g. [116])

$$
\begin{aligned}
g\left(a+\frac{1}{2}\right) & +g\left(a+\frac{3}{2}\right)+\cdots+g\left(b-\frac{1}{2}\right) \\
& =\int_{a}^{b} d t g(t)+\sum_{n=1}^{w} \frac{\left(2^{-2 n+1}-1\right) B_{2 n}}{(2 n) !}\left[g^{(2 n-1)}(m)-g^{(2 n-1)}(0)\right]+R_{2 w-1}
\end{aligned}
$$

where the remainder function is

$$
R_{w}=\frac{(-1)^{w+1}}{w !} \int_{0}^{m} d t g^{(w+1)}(t) \zeta\left(-w, t+\frac{1}{2}\right)
$$

\footnotetext{
${ }^{17}$ Recall that the summation is always done in steps of one.
} 
and $\zeta(s, q)$ is the Hurwitz zeta function. Generally, $R_{w}$ does not vanish in the $w \rightarrow \infty$ limit and, therefore, sending $w \rightarrow \infty$ and dropping $R_{w}$ in (A.7) gives a non-convergent asymptotic expansion.

For $n \geq 1$, the second terms of (A.3) and (A.6) involve the generalized harmonic number,

$$
H_{q}^{(r)}=\sum_{m=1}^{q} \frac{1}{m^{r}} .
$$

Its asymptotic expansion for large $q$ is $[117]$

$$
H_{q}^{(r)} \sim \zeta(r)-\frac{2 q+r+1}{2(r-1)(q+1)^{r}}-\frac{1}{(r-1) !} \sum_{l=1}^{\infty} \frac{(2 l+r-2) ! B_{2 l}}{(2 l) !(q+1)^{2 l+r-1}}
$$

where " $\sim$ " means an asymptotic expansion and $\zeta(s)$ is the Riemann zeta function. By expanding this in $r$ around $r=0$ and collecting the $\mathcal{O}(r)$ terms, we obtain the asymptotic expansion

$$
\sum_{m=1}^{q} \ln m \sim \frac{1}{2} \ln (2 \pi)-1-q+\left(q+\frac{1}{2}\right) \ln (q+1)+\sum_{k=1}^{\infty} \frac{B_{2 k}}{2 k(2 k-1)(q+1)^{2 k-1}}
$$

which we can use for evaluating the $n=0$ case of (A.3) and (A.6).

Applying the above formulas (A.7), (A.10) and (A.11) to (A.3) and (A.6) and massaging the resulting expression, we obtain the following asymptotic expansion:

$$
f_{2 n} \sim \begin{cases}\int_{0}^{t} d y \ln \tan \frac{\pi y}{2}+2 \sum_{l=1}^{\infty} \frac{\left(2^{-2 l+1}-1\right) B_{2 l}}{(2 l) !} \frac{\left(2 \partial_{t}\right)^{2 l-1}}{k^{2 l}} \ln \tan \frac{\pi t}{2}+\widetilde{f}_{0} & (n=0), \\ 2 \sum_{l=0}^{\infty} \frac{\left(2^{-2 l+1}-1\right) B_{2 l}}{(2 l) !} \frac{\left(2 \partial_{t}\right)^{2 n+2 l-1}}{k^{2 l}} \ln \tan \frac{\pi t}{2}+\widetilde{f}_{2 n} & (n \geq 1),\end{cases}
$$

where, for even $M$,

$$
\begin{aligned}
k \widetilde{f}_{0}= & 2 \sum_{l=1}^{\infty} \frac{\left(2^{2 l-1}-1\right) B_{2 l}}{2 l(2 l-1) M^{2 l-1}}+(2 M+1) \ln \left(1+\frac{1}{M}\right)-(M+1) \ln \left(1+\frac{2}{M}\right) \\
& +2 \sum_{l=1}^{\infty} \frac{B_{2 l}}{2 l(2 l-1)}\left[\frac{1}{(M+1)^{2 l-1}}-\frac{1}{\left(\frac{M}{2}+1\right)^{2 l-1}}\right] \\
\frac{\widetilde{f}_{2 n}}{k^{2 n-1}}= & 2 \sum_{l=0}^{\infty} \frac{2^{2 n}\left(2^{2 l-1}-1\right)(2 n+2 l-2) ! B_{2 l}}{(2 l) ! M^{2 l+2 n-1}+(2 n-2) !\left[\frac{2^{2 n}(2 M+2 n+1)}{(M+1)^{2 n}}-\frac{M+2 n+1}{\left(\frac{M}{2}+1\right)^{2 n}}\right]} \\
& +2 \sum_{l=1}^{\infty} \frac{(2 l+2 n-2) ! B_{2 l}}{(2 l) !}\left[\frac{2^{2 n}}{(M+1)^{2 l+2 n-1}}-\frac{1}{\left(\frac{M}{2}+1\right)^{2 l+2 n-1}}\right]
\end{aligned}
$$


while, for odd $M$,

$$
\begin{aligned}
k \widetilde{f}_{0}= & 2 \sum_{l=1}^{\infty} \frac{\left(2^{2 l-1}-1\right) B_{2 l}}{2 l(2 l-1) M^{2 l-1}}+M \ln \left(1+\frac{1}{M}\right)-1+2 \sum_{l=1}^{\infty} \frac{2^{2 l-1} B_{2 l}}{2 l(2 l-1)(M+1)^{2 l-1}}, \\
\frac{\widetilde{f}_{2 n}}{k^{2 n-1}}= & 2 \sum_{l=0}^{\infty} \frac{2^{2 n}\left(2^{2 l-1}-1\right)(2 n+2 l-2) ! B_{2 l}}{(2 l) ! M^{2 l+2 n-1}} \\
& +(2 n-2) ! \frac{2^{2 n}(M+2 n)}{(M+1)^{2 n}}+2 \sum_{l=1}^{\infty} \frac{2^{2 l+2 n-1}(2 l+2 n-2) ! B_{2 l}}{(2 l) !(M+1)^{2 l+2 n-1}}
\end{aligned}
$$

with $n \geq 1$. Some comments in deriving the expression (A.12) are in order. First, the first terms in (A.3), (A.6) were evaluated using the Euler-Maclaurin formula (A.7) and formally dropping the remainder function. In the resulting integrals, we defined $y \equiv 2 m / k$ and rewrote it in terms of $y$-integrals. For $n \geq 1$, the integral can be trivially integrated to give the $l=0$ term in (A.12). Furthermore, we split $\ln \left[\left(\tan \frac{\pi y}{2}\right) /\left(\frac{\pi y}{2}\right)\right]=\ln \left[\tan \left(\frac{\pi y}{2}\right)\right]-\ln \left(\frac{\pi y}{2}\right)$ and put the ones originating from $\ln \left(\frac{\pi y}{2}\right)$ into $\widetilde{f}_{0}, \widetilde{f}_{2 n}$. Next, the second terms in (A.3), (A.6) were evaluated using the asymptotic formulas (A.10), (A.11). For odd $M$, there is no problem in directly applying the these formulas but, for even $M=2 p$, we need to use the following trick,

$$
\begin{aligned}
& \sum_{m=\frac{1}{2}}^{p-\frac{1}{2}} \ln j=-2 p \ln 2+\sum_{m=1}^{2 p} \ln m-\sum_{m=1}^{p} \ln m, \\
& \sum_{m=\frac{1}{2}}^{p-\frac{1}{2}} \frac{1}{m^{2 n}}=2^{2 n} \sum_{m=1}^{2 p} \frac{1}{m^{2 n}}-\sum_{m=1}^{p} \frac{1}{m^{2 n}}
\end{aligned}
$$

before applying the asymptotic formulas. The asymptotic formula (A.10) involves the $\zeta$ function which may look like a nuisance, but it precisely cancels the last (constant) terms in (A.3), (A.6), due to the identity

$$
\zeta(2 n)=\frac{(-1)^{n+1}(2 \pi)^{2 n} B_{2 n}}{2(2 n) !}, \quad n \geq 1
$$

Similar cancellations happen for the $\ln$ terms for $n=0$.

Actually, as we will show below, $\widetilde{f}_{0}=\widetilde{f}_{2 n}=0$. Therefore, (A.12) actually becomes

$$
f_{2 n} \sim 2 \sum_{l=0}^{\infty} \frac{\left(2^{-2 l+1}-1\right) B_{2 l}}{(2 l) !} \frac{\left(2 \partial_{t}\right)^{2 n+2 l-1}}{k^{2 l}} \ln \tan \frac{\pi t}{2} \quad(n \geq 0),
$$

where it is understood that, for $n=l=0$,

$$
\frac{1}{\partial_{t}} \ln \tan \frac{\pi t}{2}=\int_{0}^{t} d y \ln \tan \frac{\pi y}{2} .
$$

Formally carrying out the summation in (A.20), we obtain

$$
f_{2 n} \sim \frac{\left(2 \partial_{t}\right)^{2 n}}{k \sinh \frac{\partial_{t}}{k}} \ln \tan \frac{\pi t}{2} .
$$

If we substitute the expression (A.22) into (3.17) and formally perform the summation over $n$, we obtain the expression in the main text, (3.16). 
The final result (A.20) may look like the expression which we would obtain if we directly applied the Euler-Maclaurin formula (A.7) to the original expression (3.14). However, of course, the Euler-Maclaurin formula does not work in the presence of a singularity that gives a divergent integral. It is only after the above careful treatment of the singularities as we did above and the delicate cancellation of terms due to the presence of the seemingly unwanted function $R(x)$ that we arrived at the very simple expression (A.20).

Proof of $\widetilde{f}_{2 n}=\mathbf{0}$. Let us show that $\widetilde{f}_{2 n}=0$ as mentioned above. For simplicity, let us consider the case with odd $M$ and $n \geq 1$. The relevant expression is (A.16). First, because $B_{0}=1, B_{1}=-1 / 2$ and $B_{2 n+1}=0$ for $n \geq 1$, we can combine the two terms in the second line to get the following expression:

$$
\frac{\tilde{f}_{2 n}}{k^{2 n-1}}=2 \sum_{l=0}^{\infty} \frac{2^{2 n}\left(2^{2 l-1}-1\right)(2 n+2 l-2) ! B_{2 l}}{(2 l) ! M^{2 l+2 n-1}}+\sum_{l=0}^{\infty} \frac{(-1)^{l} 2^{l+2 n}(l+2 n-2) ! B_{l}}{l !(M+1)^{l+2 n-1}} .
$$

When expanded in $1 / M$, the second term is equal to

$$
\begin{aligned}
\sum_{l=0}^{\infty} \frac{(-1)^{l} 2^{l+2 n}(l+2 n-2) ! B_{l}}{l ! M^{l+2 n-1}} \sum_{p=0}^{\infty}(-1)^{p}\left(\begin{array}{c}
l+2 n+p-2 \\
p
\end{array}\right) \frac{1}{M^{p}} \\
\quad=\sum_{q=0}^{\infty} \sum_{l=0}^{q} \frac{(-1)^{q} 2^{l+2 n} !(q+2 n-2) ! B_{l}}{M^{q+2 n-1} l !(q-l) !} \quad(l+p \equiv q) \\
\quad=\sum_{q=0}^{\infty} \frac{(-1)^{q} 2^{2 n}(q+2 n-2) ! B_{l}}{M^{q+2 n-1} q !} \sum_{l=0}^{q}\left(\begin{array}{l}
q \\
l
\end{array}\right) 2^{l} B_{l} .
\end{aligned}
$$

Now, recalling the relation between the Bernoulli polynomial $B_{n}(x)$ and the Bernoulli numbers $B_{n}$,

$$
B_{n}(x)=\sum_{l=0}^{n}\left(\begin{array}{l}
n \\
l
\end{array}\right) x^{n-l} B_{l}
$$

and also the relation

$$
B_{n}\left(\frac{1}{2}\right)=\left(2^{1-n}-1\right) B_{n}
$$

we find

$$
\sum_{l=0}^{q}\left(\begin{array}{l}
q \\
l
\end{array}\right) 2^{l} B_{l}=2^{q} \sum_{l=0}^{q}\left(\begin{array}{l}
q \\
l
\end{array}\right)\left(\frac{1}{2}\right)^{q-l} B_{l}=2^{q} B_{q}\left(\frac{1}{2}\right)=2^{q}\left(2^{1-q}-1\right) B_{q} .
$$

Therefore,

$$
(\mathrm{A} .24)=\sum_{q=0}^{\infty} \frac{(-1)^{q} 2^{q+2 n}\left(2^{-q+1}-1\right)(q+2 n-2) ! B_{q}}{q ! M^{q+2 n-1}} .
$$

Because the summand vanishes for $q=1$ and because $B_{2 n+1}=0$ for $n \geq 1$, we can set $q=2 l, l \geq 0$. Then this cancels the first term in (A.23). So, we have shown $\widetilde{f}_{2 n}=0$.

In a quite similar manner, using Bernoulli polynomial/number identities, we can show that $\widetilde{f}_{0}=0$ for even $M$ and $\widetilde{f}_{0}=\widetilde{f}_{2 n}=0(n \geq 1)$ for odd $M$. 


\section{B Evaluation of the matrix integral (3.23)}

In this appendix, we would like to systematically evaluate the integral (3.23), which we write down here again for convenience:

$$
\begin{aligned}
\Psi(N ; M)_{k}=e^{-\mathcal{F}(N ; M)_{k}}= & \frac{\pi^{N(N-1)} e^{k N f_{0}}}{N ! k^{\frac{N^{2}}{2}-N}}\left[\prod_{j=1}^{N} \int_{-\infty}^{\infty} d \xi_{j}\right] \Delta(\xi)^{2} \\
& \times \exp \left[\sum_{n=1}^{\infty} \frac{(-1)^{n} f_{2 n}}{(2 n) ! k^{n-1}} \sum_{j=1}^{N} \xi_{j}^{2 n}+2 \sum_{j<m} \ln \frac{\tanh \frac{\pi\left(\xi_{j}-\xi_{m}\right)}{k^{1 / 2}}}{\frac{\pi\left(\xi_{j}-\xi_{m}\right)}{k^{1 / 2}}}\right] .
\end{aligned}
$$

Note that $\mathcal{F}$ defined here is different from the full ABJ free energy $F_{\mathrm{ABJ}}=-\ln Z_{\mathrm{ABJ}}$ which contains more terms coming from (3.7).

Because $f_{2 n}=f_{2 n}(k, t)=\mathcal{O}\left(k^{0}\right)$, we can treat the $\xi^{2}$ term in the exponential of (B.1) as the propagator and all higher power terms as interactions, and evaluate the integral perturbatively in a $1 / k$ expansion. The last term in the exponential can be written as

$$
\sum_{j<m} \ln \frac{\tanh \frac{\pi\left(\xi_{j}-\xi_{m}\right)}{k^{1 / 2}}}{\frac{\pi\left(\xi_{j}-\xi_{m}\right)}{k^{1 / 2}}}=\sum_{n=1}^{\infty} c_{2 n}\left(\frac{\pi^{2}}{k}\right)^{n} \sum_{j<m}\left(\xi_{i}-\xi_{j}\right)^{2 n}
$$

where we used the relation [115, eq. 1.518.3]

$$
\ln \frac{\tan x}{x}=\sum_{n=1}^{\infty} c_{2 n} x^{2 n}, \quad c_{2 n}=\frac{(-1)^{n+1}\left(2^{2 n-1}-1\right) 2^{2 n} B_{2 n}}{n(2 n) !} .
$$

To avoid clutter, let us use the shorthand notation

$$
\prod_{j=1}^{N} \int_{-\infty}^{\infty} d \xi_{j} \equiv \int d^{N} \xi, \quad \sum_{j=1}^{N} \xi_{j}^{n} \equiv \xi^{n}, \quad \sum_{1 \leq j<m \leq N}\left(\xi_{j}-\xi_{m}\right)^{2 n} \equiv(\Delta \xi)^{2 n} .
$$

First, note that the Gaussian integral of the quadratic term is given by

$$
\int d^{N} \xi \Delta(\xi)^{2} e^{-\frac{f_{2}}{2} \xi^{2}}=f_{2}^{-\frac{N^{2}}{2}}(2 \pi)^{\frac{N}{2}} G_{2}(N+2),
$$

where $G_{2}(N)$ is the Barnes $G$-function. For a quantity $\mathcal{O}(\xi)$, let us define its expectation value by

$$
\langle\mathcal{O}\rangle \equiv \frac{\int d^{N} \xi \Delta(\xi)^{2} e^{-\frac{f_{2}}{2} \xi^{2}} \mathcal{O}}{\int d^{N} \xi \Delta(\xi)^{2} e^{-\frac{f_{2}}{2} \xi^{2}}} .
$$

Then the integral (B.1) can be written as

$$
\begin{aligned}
e^{-\mathcal{F}(N ; M)_{k}}= & \frac{2^{\frac{N}{2}} G_{2}(N+1) \pi^{N^{2}-\frac{N}{2}} e^{k N f_{0}}}{k^{\frac{N^{2}}{2}-N} f_{2}^{\frac{N^{2}}{2}}} \\
& \times\left\langle\exp \left[\sum_{n=2}^{\infty} \frac{(-1)^{n} f_{2 n}}{(2 n) ! k^{2 n-1}} \xi^{2 n}+\sum_{n=1}^{\infty} c_{2 n}\left(\frac{\pi^{2}}{k}\right)^{n}(\Delta \xi)^{2 n}\right]\right\rangle,
\end{aligned}
$$

where we used the relation $G_{2}(z+1)=\Gamma(z) G_{2}(z)$. 
The above is sufficient for computing $\mathcal{F}(N ; M)_{k}$ in principle, but the following observation makes the computation simpler. Note that $\Delta(\xi)^{2}$ is nothing but the Fadeev-Popov determinant for going from the matrix model of an $N \times N$ Hermitian matrix $X$ to the diagonal gauge where $\xi_{j}, j=1, \ldots, N$ are the eigenvalues of $X$. So, the expectation value of $\mathcal{O}$ defined in (B.6) can be written as the expectation value in a Hermitian matrix model as

$$
\langle\mathcal{O}\rangle=\frac{\int d^{N^{2} X} e^{-\frac{f_{2}}{2} \operatorname{tr} X^{2}} \mathcal{O}}{\int d^{N^{2}} X e^{-\frac{f_{2}}{2} \operatorname{tr} X^{2}}},
$$

where $X$ is an $N \times N$ Hermitean matrix. When going from the eigenvalue basis in terms of $\xi_{j}$ back to the Hermitean matrix model, we do the following replacements in $\mathcal{O}$ :

$$
\begin{aligned}
\xi^{2 n} & =\sum_{i} \xi_{i}^{2 n} \rightarrow \operatorname{tr} X^{2 n} \\
(\Delta \xi)^{2 n} & =\sum_{i<j}\left(\xi_{i}-\xi_{j}\right)^{2 n}=\frac{1}{2} \sum_{i, j}\left(\xi_{i}-\xi_{j}\right)^{2 n}=\frac{1}{2} \sum_{i, j} \sum_{l=0}^{2 n}(-1)^{l}\left(\begin{array}{c}
2 n \\
l
\end{array}\right) \xi_{i}^{l} \xi_{j}^{2 n-l} \\
& \rightarrow \frac{1}{2} \sum_{l=0}^{2 n}(-1)^{l}\left(\begin{array}{c}
2 n \\
l
\end{array}\right) \operatorname{tr} X^{l} \operatorname{tr} X^{2 n-l} \\
& =\sum_{l=0}^{n-1}(-1)^{l}\left(\begin{array}{c}
2 n \\
l
\end{array}\right) \operatorname{tr} X^{l} \operatorname{tr} X^{2 n-l}+\frac{(-1)^{n}}{2}\left(\begin{array}{c}
2 n \\
n
\end{array}\right)\left(\operatorname{tr} X^{n}\right)^{2} \equiv(\Delta X)^{2 n}
\end{aligned}
$$

and use the contraction rule

$$
\left\langle X^{\alpha}{ }_{\beta} X^{\gamma}\right\rangle=f_{2}^{-1} \delta_{\delta}^{\alpha} \delta_{\beta}^{\gamma} .
$$

Some of the correlators computed using the matrix model diagrams are:

$$
\begin{aligned}
\left\langle\xi^{2}\right\rangle & =\left\langle\operatorname{tr} X^{2}\right\rangle=N^{2}, \quad\left\langle\left(\xi^{1}\right)^{2}\right\rangle=\left\langle(\operatorname{tr} X)^{2}\right\rangle=N, \\
\left\langle(\Delta \xi)^{2}\right\rangle & =\left\langle N \operatorname{tr} X^{2}-(\operatorname{tr} X)^{2}\right\rangle=N^{3}-N, \\
\left\langle\xi^{4}\right\rangle & =\left\langle\operatorname{tr} X^{4}\right\rangle=2 N^{3}+N, \quad\left\langle\xi^{3} \xi^{1}\right\rangle=\left\langle\operatorname{tr} X^{3} \operatorname{tr} X\right\rangle=3 N^{2}, \\
\left\langle\xi^{2} \xi^{2}\right\rangle & =\left\langle\left(\operatorname{tr} X^{2}\right)^{2}\right\rangle=N^{4}+2 N^{2}, \quad\left\langle\xi^{2}\left(\xi^{1}\right)^{2}\right\rangle=\left\langle\operatorname{tr} X^{2}(\operatorname{tr} X)^{2}\right\rangle=N^{3}+2 N, \\
\left\langle\left(\xi^{1}\right)^{4}\right\rangle & =\left\langle(\operatorname{tr} X)^{4}\right\rangle=3 N^{2}, \\
\left\langle(\Delta \xi)^{4}\right\rangle & =\left\langle N \operatorname{tr} X^{4}-4 \operatorname{tr} X^{3} \operatorname{tr} X+3\left(\operatorname{tr} X^{2}\right)^{2}\right\rangle=5 N^{4}-5 N^{2}, \\
\left\langle\left(\Delta \xi^{2}\right)^{2}\right\rangle & =\left\langle\left[N \operatorname{tr} X^{2}-(\operatorname{tr} X)^{2}\right]^{2}\right\rangle=N^{6}-N^{2}, \\
\left\langle\xi^{6}\right\rangle & =\left\langle\operatorname{tr} X^{6}\right\rangle=5 N^{4}+10 N^{2}, \quad\left\langle\xi^{4} \xi^{2}\right\rangle=2 N^{5}+9 N^{3}+4 N, \\
\left\langle\xi^{4}\left(\xi^{1}\right)^{2}\right\rangle & =2 N^{4}+13 N^{2}, \quad\left\langle\xi^{4}(\Delta \xi)^{2}\right\rangle=\left\langle\xi^{4}\left[N \operatorname{tr} X^{2}-(\operatorname{tr} X)^{2}\right]\right\rangle=2 N^{6}+7 N^{4}-9 N^{2}, \\
\left\langle\left(\xi^{4}\right)^{2}\right\rangle & =\left\langle\left(\operatorname{tr} X^{4}\right)^{2}\right\rangle=4 N^{6}+40 N^{4}+61 N^{2} .
\end{aligned}
$$

In the above expressions, we set $f_{2}=1$ for simplicity, but the correct powers of $f_{2}$ can be recovered on dimensional grounds. When computing correlators such as (B.12), diagrams get out of hand quickly as the power grows. Rather than directly dealing with diagrams, 
it is easier to assume that a given correlator is an even/odd polynomial in $N$ with certain degree, and determine the coefficients by computer for some small values of $N$.

So, in terms of the Hermitian matrix model, the "free energy" $\mathcal{F}(N ; M)_{k}$ can be computed as follows:

$$
\begin{aligned}
\mathcal{F}(N ; M)_{k}= & -k N f_{0}+\frac{N^{2}}{2} \ln \frac{k f_{2}}{\pi}-\frac{N}{2} \ln \frac{2 k^{2}}{\pi}-\ln G_{2}(N+1) \\
& +\left\langle\exp \left[\sum_{n=2}^{\infty} \frac{(-1)^{n} f_{2 n}}{(2 n) ! k^{2 n-1}} \operatorname{tr} X^{2 n}+\sum_{n=1}^{\infty} c_{2 n}\left(\frac{\pi^{2}}{k}\right)^{n}(\Delta X)^{2 n}\right]-1\right\rangle_{\text {conn }},
\end{aligned}
$$

where \langle\rangle$_{\text {conn }}$ means the connected part; for example,

$$
\left\langle\left(\operatorname{tr} X^{2}\right)^{2}\right\rangle_{\text {conn }}=\left\langle\left(\operatorname{tr} X^{2}\right)^{2}\right\rangle-\left\langle\operatorname{tr} X^{2}\right\rangle^{2} .
$$

Carrying out the diagram expansion in (B.13) to a few orders and using the large $k$ expansion of $f_{2 n}(k, t)$ given in (3.18), we obtain the following large $k$ expansion for $\mathcal{F}(N ; M)_{k}$ :

$$
\begin{aligned}
& \mathcal{F}(N ; M)_{k} \\
& =\frac{2 k N}{\pi} \mathcal{I}\left(\frac{\pi t}{2}\right)+\frac{N^{2}}{2} \ln \frac{4 k}{\pi \sin (\pi t)}-\frac{N}{2} \ln \frac{2 k^{2}}{\pi}-\ln G_{2}(N+1) \\
& -\frac{\pi N\left(2 N^{2}-1\right)}{48 \sin (\pi t) k}[3 \cos (2 \pi t)+1] \\
& -\frac{\pi^{2} N^{2}}{2304 \sin ^{2}(\pi t) k^{2}}\left[\left(17 N^{2}+1\right) \cos (4 \pi t)+4\left(11 N^{2}-29\right) \cos (2 \pi t)-157 N^{2}+211\right] \\
& -\frac{\pi^{3} N}{552960 \sin ^{3}(\pi t) k^{3}}\left[\left(674 N^{4}+250 N^{2}+201\right) \cos (6 \pi t)-6\left(442 N^{4}+690 N^{2}-427\right) \cos (4 \pi t)\right. \\
& \left.\quad+3\left(2282 N^{4}+3490 N^{2}-3635\right) \cos (2 \pi t)+4348 N^{4}-21940 N^{2}+12750\right] \\
& -\frac{\pi^{4} N^{2}}{22118400 \sin ^{4}(\pi t) k^{4}}\left[\left(6223 N^{4}+8330 N^{2}+2997\right) \cos (8 \pi t)\right. \\
& \quad-8\left(3983 N^{4}+6730 N^{2}-363\right) \cos (6 \pi t)+20\left(3797 N^{4}+1870 N^{2}+1623\right) \cos (4 \pi t) \\
& \left.\quad-8\left(22249 N^{4}-44410 N^{2}+37011\right) \cos (2 \pi t)-56627 N^{4}+113630 N^{2}-18753\right] \\
& +\mathcal{O}\left(k^{-5}\right) .
\end{aligned}
$$

Rewriting this as a large $M$ expansion gives eq. (3.25) presented in the main text. 
Open Access. This article is distributed under the terms of the Creative Commons Attribution License (CC-BY 4.0), which permits any use, distribution and reproduction in any medium, provided the original author(s) and source are credited.

\section{References}

[1] D.J. Gross, High-Energy Symmetries of String Theory, Phys. Rev. Lett. 60 (1988) 1229 [INSPIRE].

[2] D.J. Gross and P.F. Mende, String Theory Beyond the Planck Scale, Nucl. Phys. B 303 (1988) 407 [INSPIRE].

[3] J.J. Atick and E. Witten, The Hagedorn Transition and the Number of Degrees of Freedom of String Theory, Nucl. Phys. B 310 (1988) 291 [INSPIRE].

[4] M.A. Vasiliev, More on equations of motion for interacting massless fields of all spins in (3+1)-dimensions, Phys. Lett. B 285 (1992) 225 [INSPIRE].

[5] E.S. Fradkin and M.A. Vasiliev, On the Gravitational Interaction of Massless Higher Spin Fields, Phys. Lett. B 189 (1987) 89 [inSPIRE].

[6] E.S. Fradkin and M.A. Vasiliev, Cubic Interaction in Extended Theories of Massless Higher Spin Fields, Nucl. Phys. B 291 (1987) 141 [INSPIRE].

[7] M.A. Vasiliev, Higher spin gauge theories: Star product and AdS space, hep-th/9910096 [INSPIRE].

[8] M.A. Vasiliev, Higher spin gauge theories in four-dimensions, three-dimensions and two-dimensions, Int. J. Mod. Phys. D 5 (1996) 763 [hep-th/9611024] [INSPIRE].

[9] S. Weinberg, Photons and Gravitons in s Matrix Theory: Derivation of Charge Conservation and Equality of Gravitational and Inertial Mass, Phys. Rev. 135 (1964) B1049 [INSPIRE].

[10] S.R. Coleman and J. Mandula, All Possible Symmetries of the S Matrix, Phys. Rev. 159 (1967) 1251 [INSPIRE].

[11] C. Aragone and S. Deser, Consistency Problems of Hypergravity, Phys. Lett. B 86 (1979) 161 [INSPIRE].

[12] S. Weinberg and E. Witten, Limits on Massless Particles, Phys. Lett. B 96 (1980) 59 [INSPIRE].

[13] M. Porrati, Universal Limits on Massless High-Spin Particles, Phys. Rev. D 78 (2008) 065016 [arXiv: 0804.4672] [INSPIRE].

[14] I.R. Klebanov and A.M. Polyakov, AdS dual of the critical $O(N)$ vector model, Phys. Lett. B 550 (2002) 213 [hep-th/0210114] [INSPIRE].

[15] S. Giombi and X. Yin, Higher Spin Gauge Theory and Holography: The Three-Point Functions, JHEP 09 (2010) 115 [arXiv:0912.3462] [INSPIRE].

[16] S. Giombi and X. Yin, The Higher Spin/Vector Model Duality, J. Phys. A 46 (2013) 214003 [arXiv: 1208.4036] [INSPIRE].

[17] S. Giombi and I.R. Klebanov, One Loop Tests of Higher Spin AdS/CFT, JHEP 12 (2013) 068 [arXiv: 1308.2337] [INSPIRE].

[18] S. Giombi, I.R. Klebanov, S.S. Pufu, B.R. Safdi and G. Tarnopolsky, AdS Description of Induced Higher-Spin Gauge Theory, JHEP 10 (2013) 016 [arXiv:1306.5242] [INSPIRE]. 
[19] A.A. Tseytlin, On partition function and Weyl anomaly of conformal higher spin fields, Nucl. Phys. B 877 (2013) 598 [arXiv: 1309. 0785] [INSPIRE].

[20] S. Giombi, I.R. Klebanov and B.R. Safdi, Higher Spin AdS $S_{d+1} / C F T_{d}$ at One Loop, Phys. Rev. D 89 (2014) 084004 [arXiv: 1401.0825] [InSPIRE].

[21] A. Jevicki, K. Jin and J. Yoon, $1 / N$ and loop corrections in higher spin $A d S_{4} / C F T_{3}$ duality, Phys. Rev. D 89 (2014) 085039 [arXiv: 1401.3318] [INSPIRE].

[22] A. Jevicki, K. Jin and Q. Ye, Perturbative and Non-Perturbative Aspects in Vector Model/Higher Spin Duality, J. Phys. A 46 (2013) 214005 [arXiv:1212.5215] [INSPIRE].

[23] R. de Mello Koch, A. Jevicki, K. Jin and J.P. Rodrigues, $A d S_{4} / C F T_{3}$ Construction from Collective Fields, Phys. Rev. D 83 (2011) 025006 [arXiv:1008.0633] [InSPIRE].

[24] A. Jevicki, K. Jin and Q. Ye, Collective Dipole Model of AdS/CFT and Higher Spin Gravity, J. Phys. A 44 (2011) 465402 [arXiv:1106.3983] [inSPIRE].

[25] R. de Mello Koch, A. Jevicki, J.P. Rodrigues and J. Yoon, Holography as a Gauge Phenomenon in Higher Spin Duality, JHEP 01 (2015) 055 [arXiv:1408.1255] [INSPIRE].

[26] R. de Mello Koch, A. Jevicki, J.P. Rodrigues and J. Yoon, Canonical Formulation of $O(N)$ Vector/Higher Spin Correspondence, J. Phys. A 48 (2015) 105403 [arXiv:1408.4800] [INSPIRE].

[27] M.R. Gaberdiel and R. Gopakumar, An AdS $S_{3}$ Dual for Minimal Model CFTs, Phys. Rev. D 83 (2011) 066007 [arXiv: 1011.2986] [INSPIRE].

[28] M.R. Gaberdiel and R. Gopakumar, Minimal Model Holography, J. Phys. A 46 (2013) 214002 [arXiv: 1207.6697] [INSPIRE].

[29] M.R. Gaberdiel and R. Gopakumar, Triality in Minimal Model Holography, JHEP 07 (2012) 127 [arXiv: 1205.2472] [INSPIRE].

[30] M.R. Gaberdiel and C. Vollenweider, Minimal Model Holography for $\mathrm{SO}(2 N)$, JHEP 08 (2011) 104 [arXiv: 1106.2634] [INSPIRE].

[31] M. Beccaria, C. Candu, M.R. Gaberdiel and M. Groher, N=1 extension of minimal model holography, JHEP 07 (2013) 174 [arXiv: 1305.1048] [INSPIRE].

[32] M.R. Gaberdiel and R. Gopakumar, Large $N=4$ Holography, JHEP 09 (2013) 036 [arXiv: 1305.4181] [INSPIRE].

[33] M.R. Gaberdiel, R. Gopakumar and A. Saha, Quantum $W$-symmetry in $A d S_{3}$, JHEP 02 (2011) 004 [arXiv: 1009.6087] [InSPIRE].

[34] M.R. Gaberdiel and T. Hartman, Symmetries of Holographic Minimal Models, JHEP 05 (2011) 031 [arXiv:1101.2910] [INSPIRE].

[35] M.R. Gaberdiel, R. Gopakumar, T. Hartman and S. Raju, Partition Functions of Holographic Minimal Models, JHEP 08 (2011) 077 [arXiv:1106.1897] [INSPIRE].

[36] A. Castro, R. Gopakumar, M. Gutperle and J. Raeymaekers, Conical Defects in Higher Spin Theories, JHEP 02 (2012) 096 [arXiv:1111.3381] [INSPIRE].

[37] T. Creutzig, Y. Hikida and P.B. Rønne, Higher spin AdS $S_{3}$ supergravity and its dual CFT, JHEP 02 (2012) 109 [arXiv:1111.2139] [InSPIRE].

[38] T. Creutzig, Y. Hikida and P.B. Rønne, $N=1$ supersymmetric higher spin holography on $A d S_{3}, J H E P 02$ (2013) 019 [arXiv:1209.5404] [INSPIRE].

[39] T. Creutzig, Y. Hikida and P.B. Rønne, Extended higher spin holography and Grassmannian models, JHEP 11 (2013) 038 [arXiv:1306.0466] [INSPIRE]. 
[40] Y. Hikida, Conical defects and $N=2$ higher spin holography, JHEP 08 (2013) 127 [arXiv: 1212.4124] [INSPIRE].

[41] C. Candu and M.R. Gaberdiel, Duality in $N=2$ Minimal Model Holography, JHEP 02 (2013) 070 [arXiv: 1207.6646] [inSPIRE].

[42] M. Gutperle and P. Kraus, Higher Spin Black Holes, JHEP 05 (2011) 022 [arXiv: 1103.4304] [INSPIRE].

[43] M. Ammon, M. Gutperle, P. Kraus and E. Perlmutter, Black holes in three dimensional higher spin gravity: A review, J. Phys. A 46 (2013) 214001 [arXiv:1208.5182] [INSPIRE].

[44] M. Ammon, M. Gutperle, P. Kraus and E. Perlmutter, Spacetime Geometry in Higher Spin Gravity, JHEP 10 (2011) 053 [arXiv:1106.4788] [INSPIRE].

[45] P. Kraus and E. Perlmutter, Partition functions of higher spin black holes and their CFT duals, JHEP 11 (2011) 061 [arXiv:1108.2567] [InSPIRE].

[46] C.-M. Chang and X. Yin, Higher Spin Gravity with Matter in AdS $S_{3}$ and Its CFT Dual, JHEP 10 (2012) 024 [arXiv:1106.2580] [INSPIRE].

[47] K. Papadodimas and S. Raju, Correlation Functions in Holographic Minimal Models, Nucl. Phys. B 856 (2012) 607 [arXiv:1108.3077] [INSPIRE].

[48] M. Ammon, P. Kraus and E. Perlmutter, Scalar fields and three-point functions in D = 3 higher spin gravity, JHEP 07 (2012) 113 [arXiv:1111.3926] [INSPIRE].

[49] T. Creutzig, Y. Hikida and P.B. Ronne, Three point functions in higher spin AdS 3 supergravity, JHEP 01 (2013) 171 [arXiv:1211.2237] [INSPIRE].

[50] H. Moradi and K. Zoubos, Three-Point Functions in $N=2$ Higher-Spin Holography, JHEP 04 (2013) 018 [arXiv: 1211.2239] [INSPIRE].

[51] E. Hijano, P. Kraus and E. Perlmutter, Matching four-point functions in higher spin $A d S_{3} / C F T_{2}$, JHEP 05 (2013) 163 [arXiv: 1302.6113] [INSPIRE].

[52] M.R. Gaberdiel and R. Gopakumar, Higher Spins \& Strings, JHEP 11 (2014) 044 [arXiv: 1406.6103] [INSPIRE].

[53] M.R. Gaberdiel and R. Gopakumar, Stringy Symmetries and the Higher Spin Square, J. Phys. A 48 (2015) 185402 [arXiv:1501.07236] [inSPIRE].

[54] C.-M. Chang, S. Minwalla, T. Sharma and X. Yin, ABJ Triality: from Higher Spin Fields to Strings, J. Phys. A 46 (2013) 214009 [arXiv:1207.4485] [InSPIRE].

[55] O. Aharony, O. Bergman and D.L. Jafferis, Fractional M2-branes, JHEP 11 (2008) 043 [arXiv: 0807.4924] [INSPIRE].

[56] O. Aharony, O. Bergman, D.L. Jafferis and J. Maldacena, $N=6$ superconformal Chern-Simons-matter theories, M2-branes and their gravity duals, JHEP 10 (2008) 091 [arXiv:0806.1218] [INSPIRE].

[57] O. Aharony, A. Hashimoto, S. Hirano and P. Ouyang, D-brane Charges in Gravitational Duals of 2+1 Dimensional Gauge Theories and Duality Cascades, JHEP 01 (2010) 072 [arXiv:0906.2390] [INSPIRE].

[58] O. Bergman and S. Hirano, Anomalous radius shift in $A d S_{4} / C F T_{3}, J H E P ~ 07$ (2009) 016 [arXiv: 0902 .1743] [INSPIRE].

[59] E. Sezgin and P. Sundell, Higher spin $N=8$ supergravity, JHEP 11 (1998) 016 [hep-th/9805125] [INSPIRE]. 
[60] E. Sezgin and P. Sundell, Higher spin $N=8$ supergravity in $A d S_{4}$, hep-th/9903020 [INSPIRE].

[61] R. Gopakumar, From free fields to AdS, Phys. Rev. D 70 (2004) 025009 [hep-th/0308184] [INSPIRE].

[62] R. Gopakumar, From free fields to AdS. II, Phys. Rev. D 70 (2004) 025010 [hep-th/0402063] [INSPIRE].

[63] R. Gopakumar, From free fields to AdS. III, Phys. Rev. D 72 (2005) 066008 [hep-th/0504229] [INSPIRE].

[64] R. Gopakumar, Free field theory as a string theory?, Comptes Rendus Physique 5 (2004) 1111 [hep-th/0409233] [INSPIRE].

[65] K. Furuuchi, From free fields to AdS: Thermal case, Phys. Rev. D 72 (2005) 066009 [hep-th/0505148] [INSPIRE].

[66] N. Beisert, M. Bianchi, J.F. Morales and H. Samtleben, Higher spin symmetry and $N=4$ SYM, JHEP 07 (2004) 058 [hep-th/0405057] [INSPIRE].

[67] S.S. Razamat, On a worldsheet dual of the Gaussian matrix model, JHEP 07 (2008) 026 [arXiv:0803.2681] [INSPIRE].

[68] A. Kapustin, B. Willett and I. Yaakov, Exact Results for Wilson Loops in Superconformal Chern-Simons Theories with Matter, JHEP 03 (2010) 089 [arXiv:0909.4559] [INSPIRE].

[69] N. Hama, K. Hosomichi and S. Lee, SUSY Gauge Theories on Squashed Three-Spheres, JHEP 05 (2011) 014 [arXiv:1102.4716] [INSPIRE].

[70] V. Pestun, Localization of gauge theory on a four-sphere and supersymmetric Wilson loops, Commun. Math. Phys. 313 (2012) 71 [arXiv:0712.2824] [INSPIRE].

[71] M. Mariño, Lectures on localization and matrix models in supersymmetric Chern-Simons-matter theories, J. Phys. A 44 (2011) 463001 [arXiv:1104.0783] [inSPIRE].

[72] N. Drukker, M. Mariño and P. Putrov, Nonperturbative aspects of ABJM theory, JHEP 11 (2011) 141 [arXiv: 1103.4844] [INSPIRE].

[73] M. Mariño and P. Putrov, ABJM theory as a Fermi gas, J. Stat. Mech. 1203 (2012) P03001 [arXiv: 1110.4066] [INSPIRE].

[74] H. Fuji, S. Hirano and S. Moriyama, Summing Up All Genus Free Energy of ABJM Matrix Model, JHEP 08 (2011) 001 [arXiv:1106.4631] [INSPIRE].

[75] Y. Hatsuda, S. Moriyama and K. Okuyama, Instanton Bound States in ABJM Theory, JHEP 05 (2013) 054 [arXiv: 1301.5184] [InSPIRE].

[76] Y. Hatsuda, M. Mariño, S. Moriyama and K. Okuyama, Non-perturbative effects and the refined topological string, JHEP 09 (2014) 168 [arXiv:1306.1734] [INSPIRE].

[77] F. Calvo and M. Mariño, Membrane instantons from a semiclassical TBA, JHEP 05 (2013) 006 [arXiv: 1212.5118] [INSPIRE].

[78] J. Kallen and M. Mariño, Instanton effects and quantum spectral curves, Annales Henri Poincaré 17 (2016) 1037 [arXiv: 1308.6485] [INSPIRE].

[79] Y. Hatsuda, Spectral zeta function and non-perturbative effects in ABJM Fermi-gas, JHEP 11 (2015) 086 [arXiv: 1503.07883] [INSPIRE].

[80] A. Grassi, Y. Hatsuda and M. Mariño, Quantization conditions and functional equations in ABJ(M) theories, J. Phys. A 49 (2016) 115401 [arXiv:1410.7658] [InSPIRE]. 
[81] S. Codesido, A. Grassi and M. Mariño, Exact results in $\mathcal{N}=8$ Chern-Simons-matter theories and quantum geometry, JHEP 07 (2015) 011 [arXiv: 1409.1799] [INSPIRE].

[82] J. Kallen, The spectral problem of the ABJ Fermi gas, JHEP 10 (2015) 029 [arXiv:1407.0625] [INSPIRE].

[83] A. Klemm, M. Mariño, M. Schiereck and M. Soroush, Aharony-Bergman-Jafferis-Maldacena Wilson loops in the Fermi gas approach, Z. Naturforsch. A 68 (2013) 178 [arXiv:1207.0611] [INSPIRE].

[84] A. Grassi, J. Kallen and M. Mariño, The topological open string wavefunction, Commun. Math. Phys. 338 (2015) 533 [arXiv: 1304.6097] [InSPIRE].

[85] Y. Hatsuda, M. Honda, S. Moriyama and K. Okuyama, ABJM Wilson Loops in Arbitrary Representations, JHEP 10 (2013) 168 [arXiv:1306.4297] [INSPIRE].

[86] S. Hirano, K. Nii and M. Shigemori, ABJ Wilson loops and Seiberg duality, PTEP 2014 (2014) 113B04 [arXiv:1406.4141] [INSPIRE].

[87] M. Mariño and P. Putrov, Interacting fermions and $N=2$ Chern-Simons-matter theories, JHEP 11 (2013) 199 [arXiv:1206.6346] [INSPIRE].

[88] M. Honda and S. Moriyama, Instanton Effects in Orbifold ABJM Theory, JHEP 08 (2014) 091 [arXiv: 1404.0676] [INSPIRE].

[89] S. Moriyama and T. Nosaka, Partition Functions of Superconformal Chern-Simons Theories from Fermi Gas Approach, JHEP 11 (2014) 164 [arXiv:1407.4268] [INSPIRE].

[90] S. Moriyama and T. Nosaka, ABJM membrane instanton from a pole cancellation mechanism, Phys. Rev. D 92 (2015) 026003 [arXiv:1410.4918] [INSPIRE].

[91] S. Moriyama and T. Nosaka, Exact Instanton Expansion of Superconformal Chern-Simons Theories from Topological Strings, JHEP 05 (2015) 022 [arXiv: 1412.6243] [INSPIRE].

[92] H. Awata, S. Hirano and M. Shigemori, The Partition Function of ABJ Theory, PTEP 2013 (2013) 053B04 [arXiv: 1212.2966] [INSPIRE].

[93] M. Honda, Direct derivation of "mirror" ABJ partition function, JHEP 12 (2013) 046 [arXiv:1310.3126] [INSPIRE].

[94] N. Drukker, M. Mariño and P. Putrov, From weak to strong coupling in ABJM theory, Commun. Math. Phys. 306 (2011) 511 [arXiv:1007.3837] [INSPIRE].

[95] J. Maldacena and A. Zhiboedov, Constraining conformal field theories with a slightly broken higher spin symmetry, Class. Quant. Grav. 30 (2013) 104003 [arXiv:1204.3882] [InSPIRE].

[96] N. Boulanger and P. Sundell, An action principle for Vasiliev's four-dimensional higher-spin gravity, J. Phys. A 44 (2011) 495402 [arXiv:1102.2219] [INSPIRE].

[97] N. Boulanger, N. Colombo and P. Sundell, A minimal BV action for Vasiliev's four-dimensional higher spin gravity, JHEP 10 (2012) 043 [arXiv: 1205.3339] [INSPIRE].

[98] N. Doroud and L. Smolin, An Action for higher spin gauge theory in four dimensions, arXiv:1102.3297 [INSPIRE].

[99] M.A. Vasiliev, Consistent Equations for Interacting Massless Fields of All Spins in the First Order in Curvatures, Annals Phys. 190 (1989) 59 [INSPIRE].

[100] M. Honda, Identification of Bulk coupling constant in Higher Spin/ABJ correspondence, JHEP 08 (2015) 110 [arXiv:1506.00781] [INSPIRE]. 
[101] O. Aharony, G. Gur-Ari and R. Yacoby, Correlation Functions of Large-N Chern-Simons-Matter Theories and Bosonization in Three Dimensions, JHEP 12 (2012) 028 [arXiv: 1207.4593] [INSPIRE].

[102] A. Giveon and D. Kutasov, Seiberg Duality in Chern-Simons Theory, Nucl. Phys. B 812 (2009) 1 [arXiv:0808.0360] [INSPIRE].

[103] O. Aharony, S. Giombi, G. Gur-Ari, J. Maldacena and R. Yacoby, The Thermal Free Energy in Large-N Chern-Simons-Matter Theories, JHEP 03 (2013) 121 [arXiv:1211.4843] [INSPIRE].

[104] S. Matsumoto and S. Moriyama, ABJ Fractional Brane from ABJM Wilson Loop, JHEP 03 (2014) 079 [arXiv: 1310.8051] [INSPIRE].

[105] M. Honda and K. Okuyama, Exact results on ABJ theory and the refined topological string, JHEP 08 (2014) 148 [arXiv: 1405.3653] [InSPIRE].

[106] F. Benini, C. Closset and S. Cremonesi, Chiral flavors and M2-branes at toric CY4 singularities, JHEP 02 (2010) 036 [arXiv:0911.4127] [INSPIRE].

[107] A. Kapustin, B. Willett and I. Yaakov, Nonperturbative Tests of Three-Dimensional Dualities, JHEP 10 (2010) 013 [arXiv: 1003.5694] [INSPIRE].

[108] R.K. Gupta and S. Lal, Partition Functions for Higher-Spin theories in AdS, JHEP 07 (2012) 071 [arXiv: 1205.1130] [inSPIRE].

[109] E. Sezgin and P. Sundell, Supersymmetric Higher Spin Theories, J. Phys. A 46 (2013) 214022 [arXiv: 1208.6019] [INSPIRE].

[110] J. Maldacena and A. Zhiboedov, Constraining Conformal Field Theories with A Higher Spin Symmetry, J. Phys. A 46 (2013) 214011 [arXiv:1112.1016] [inSPIRE].

[111] R. Camporesi and A. Higuchi, Arbitrary spin effective potentials in anti-de Sitter space-time, Phys. Rev. D 47 (1993) 3339 [InSPIRE].

[112] R. Camporesi and A. Higuchi, Stress energy tensors in anti-de Sitter space-time, Phys. Rev. D 45 (1992) 3591 [INSPIRE].

[113] R. Camporesi, The Spinor heat kernel in maximally symmetric spaces, Commun. Math. Phys. 148 (1992) 283 [INSPIRE].

[114] S. Giombi, S. Minwalla, S. Prakash, S.P. Trivedi, S.R. Wadia and X. Yin, Chern-Simons Theory with Vector Fermion Matter, Eur. Phys. J. C 72 (2012) 2112 [arXiv:1110.4386] [INSPIRE].

[115] I.S. Gradshteyn and I.M. Ryzhik, Table of Integrals, Series and Products, Academic Press (2007).

[116] J.N. Lyness and B.W. Ninham, Numerical quadrature and asymptotic expansions, Math. Comput. 21 (1967) 162.

[117] http://functions.wolfram.com/GammaBetaErf/HarmonicNumber2/06/02/. 